\title{
Which massive stars are gamma-ray burst progenitors?
}

\author{
J. Petrovic ${ }^{1,2}$, N. Langer ${ }^{1}$, S.-C. Yoon ${ }^{1,3}$, and A. Heger ${ }^{4,5}$ \\ 1 Sterrenkundig Instituut, Utrecht University, Princetonplein 5, 3584 CC Utrecht, The Netherlands \\ e-mail: petrovic@astro.ru.nl \\ 2 Astronomical Institute, Radboud Universiteit Nijmegen, Toernooiveld 1, 6525 ED Nijmegen, The Netherlands \\ 3 Astronomical Institute "Anton Pannekoek", Amsterdam University Kruislaan 403, 1098 SJ Amsterdam, The Netherlands \\ 4 Theoretical Astrophysics Group, T-6, MS B227, Los Alamos National Laboratory, Los Alamos, NM 87545, USA \\ 5 Enrico Fermi Institute, The University of Chicago, 5640 S. Ellis Ave, Chicago, IL 60637, USA
}

Received 15 December 2004 / Accepted 3 February 2005

\begin{abstract}
The collapsar model for gamma-ray bursts requires three essential ingredients: a massive core, removal of the hydrogen envelope, and enough angular momentum in the core. We study current massive star evolution models of solar metallicity to determine which massive star physics is capable of producing these ingredients. In particular, we investigate the role of hydrodynamic and magnetic internal angular momentum transport and binary mass and angular momentum transfer. We follow the evolution of rotating single stars and of binary systems that include rotational processes for both stars. Neglecting magnetic fields, we show that the cores of massive single stars can maintain a high specific angular momentum $\left(j \sim 10^{17} \mathrm{~cm}^{2} \mathrm{~s}^{-1}\right)$ when evolved with the assumption that mean molecular weight gradient suppresses rotational mixing processes. In binary systems that undergo mass transfer during core hydrogen burning the mass receiving star accretes large amounts of high angular momentum material, leading to a spin-up of the core. We find, however, that this merely compensates for the tidal angular momentum loss due to spin-orbit coupling, which leads to synchronous rotation before the mass transfer event. Therefore the resulting cores do not rotate faster than in single stars. We show that some accreting stars become Wolf-Rayet stars at core helium exhaustion and form CO-cores that are massive enough to form a black hole. We also present models that include magnetic fields generated by differential rotation and we consider the internal angular momentum transport by magnetic torques. Though magnetic single star models are known to develop rather slowly rotating cores with specific angular momenta at the end of the evolution close to those in observed young pulsars $\left(j \sim 10^{14} \mathrm{~cm}^{2} \mathrm{~s}^{-1}\right)$, we investigate the capability of magnetic torques to efficiently pump angular momentum into the cores of accreting stars. Despite our finding that this mechanism works, the magnetic coupling of core and envelope after the accreting star ends core hydrogen burning leads to slower rotation $\left(j \sim 10^{15-16} \mathrm{~cm}^{2} \mathrm{~s}^{-1}\right)$ than in the non-magnetic case. We conclude that our binary models without magnetic fields can reproduce stellar cores with a high enough specific angular momentum $\left(j \geq 3 \times 10^{16} \mathrm{~cm}^{2} \mathrm{~s}^{-1}\right)$ to produce a collapsar and a GRB. If magnetic torques are included, however, GRBs at near solar metallicity need to be produced in rather exotic binary channels, or current dynamo model overestimates the magnetic torques. But then the problem is that significant angular momentum loss from the iron core either during core collapse or from the proto-neutron star would be required.
\end{abstract}

Key words. stars: evolution - stars: binaries: close - gamma rays: bursts - stars: rotation - stars: magnetic fields

\section{Introduction}

From studies of host galaxies of gamma-ray bursts it was concluded that they occur in or close to star forming regions. Several of them are associated with a very energetic variety of type Ic supernova with broad lines ("hypernova", Price et al. 2002; Hjorth et al. 2003), thought to display the explosion of a massive Wolf-Rayet star. And finally, their afterglows show signatures of the shaping of the circumstellar medium by a massive progenitor star (van Marle et al. 2004). The most widely used model for GRB production in the context of black hole formation in a massive single star is the so called collapsar model (Woosley 1993a).
A collapsar is a massive $\left(M \gtrsim 35-40 M_{\odot}\right.$, Fryer 1999) rotating star whose core collapses to form a black hole (Woosley 1993b; MacFadyen \& Woosley 1999). If the collapsing core has enough angular momentum $\left(j \geq 3 \times 10^{16} \mathrm{~cm}^{2} \mathrm{~s}^{-1}\right.$, MacFadyen \& Woosley 1999) an accretion disk is formed around the black hole. The accretion of the rest of the core at accretion rates up to $0.1 M_{\odot} \mathrm{s}^{-1}$ by the newly-formed black hole is thought to be capable of producing a collimated highly relativistic outflow. This releases large amounts of energy $\left(\sim 10^{51} \mathrm{erg} \mathrm{s}^{-1}\right)$ some of which is deposited in the low density rotation axis of the star. In case the star has no hydrogen envelope, i.e., has a light crossing time which is less or comparable to the duration of the central accretion (about 10s), a GRB accompanied by a type $\mathrm{Ib} / \mathrm{c}$ supernova may be produced. 
The collapsar models for gamma-ray bursts thus need three essential ingredients: a massive core, loss of the hydrogen envelope, and sufficient angular momentum to form an accretion disk.

Heger et al. (2000b) have calculated models of a $25 M_{\odot}$ star that could form a black hole by fallback (SN explosion occurs while the core forms a neutron star, but so much matter fails to escape and falls back onto the neutron star that it turns into a black hole). This star ends its life as a red supergiant with an iron core of $1.9 M_{\odot}$, a helium core of $8.06 M_{\odot}$, and a low density envelope of $6.57 M_{\odot}$. They found that this star has sufficient angular momentum to form an accretion disk around the black hole which may lead to an asymmetric, jet-driven supernovae, or in the case that the star lost its hydrogen envelope, even a GRB can result. The rotating pre-supernova models of Heger et al. (2000a) predicted, for the mass range $10 . .20 M_{\odot}$, an iron core angular momentum barely sufficient for the GRB production model through collapsar, with a trend of decreasing final specific angular momentum for larger initial mass. Heger et al. (2000a) showed also that specific angular momentum of the stellar core depends significantly on the inhibiting effect of the mean molecular weight gradient on rotational mixing processes.

Only if composition is an efficient barrier for rotational mixing and transport of angular momentum, it prevents the core from losing most of its angular momentum during the evolution. Finally, the rotating pre-supernova models of (Hirschi et al. 2004) predict about twice as much final core angular momentum as the models of Heger et al. (2000a).

These calculations left out the influence of a magnetic field which can significantly alter the angular momentum transport processes in the star, for example slowing down the helium core of the star during the red supergiant phase (Spruit \& Phinney 1998). Single star pre-supernova models including angular momentum transport by magnetic torques, using the improved dynamo model of Spruit (2002), have been produced by Heger et al. (2004a) and Heger et al. (2004b), with a clear result: although these models predict neutron star spins in the range displayed by young pulsars, the amount of angular momentum in their cores is one to two orders of magnitude less than what is required by the collapsar model of GRB production. A possible conclusion therefore might be that, if the dynamo model of Spruit (2002) is qualitatively right, massive single stars can not form GRBs within the collapsar model.

Only one in one hundred collapsing massive stars needs to produce, however, a GRB in order to explain their frequency. It seems therefore legitimate to explore collapsars in the frame of massive close binary evolution. Wellstein (2001) and Petrovic et al. (2004) showed that during the mass transfer phase in a binary system, the secondary (accreting) star can spin up to close to critical rotation, i.e., surface layers of this star can gain large amounts of angular momentum. This angular momentum can be transported inward and increase the rotation rate of the stellar core. In this case, one may expect that the effects of magnetic fields would be helpful in spinning up the core, and that faster rotating cores might be produced than in the non-magnetic models.
In this paper, we investigate four different types of rotating massive star models: single and binary models, both with and without magnetic fields included. In Sect. 2 we briefly explain our numerical methods and physical assumptions, and in Sect. 3 we explore non-magnetic single and binary stars. In Sect. 4 we present our magnetic models, first single stars then binary evolution models, and in Sect. 5 we summarize our conclusions.

\section{Computational method}

We compute detailed evolutionary rotating models of nonmagnetic $20 M_{\odot}$ and $42 M_{\odot}$ single stars, and of a $42 M_{\odot}$ single star with magnetic field included. Then we calculate the evolution of a non-magnetic and a magnetic binary system with rotating components of $M_{1, \text { in }}=56 M_{\odot}$ and $M_{2, \text { in }}=33 M_{\odot}$ and an orbital period $p_{\text {in }}=6$ days. We also compute models of a rotating $33 M_{\odot}$ star that accretes matter at the end of its main sequence evolution, for the non-magnetic and magnetic cases.

We use the evolutionary code developed by Braun (1998) on the basis of an implicit hydrodynamic stellar evolution code for single stars (Langer 1991, 1998).

The treatment of convection and semiconvection have been described in Langer (1991) and Braun \& Langer (1995). Changes in chemical composition are computed using a nuclear network including pp chains, the CNO-cycle, and the major helium, carbon, neon and oxygen burning reactions. More details are given in Wellstein \& Langer (1999) and Wellstein et al. (2001). For all models, a metallicity $Z=0.02$ is adopted and we use the OPAL opacities (Iglesias \& Rogers 1996). The abundance ratios of the isotopes are chosen to have the solar meteoritic abundance ratios according to Noels \& Grevesse (1993). The change of the orbital period due to mass transfer and stellar wind mass loss is computed according to Podsiadlowski et al. (1992), with a specific angular momentum of the stellar wind material calculated according to Brookshaw \& Tavani (1993). The influence of the centrifugal force in the rotating models is implemented according to Kippenhahn \& Thomas (1970).

Stellar wind mass loss for $\mathrm{O}$ stars is calculated according to Kudritzki et al. (1989). For hydrogen poor stars $\left(X_{\mathrm{s}}<0.4\right)$ we used a relation based on the empirical mass loss rates of Wolf-Rayet stars derived by Hamann et al. (1995):

$\log \left(\dot{M}_{\mathrm{WR}} / M_{\odot} \mathrm{yr}^{-1}\right)=-11.95+1.5 \log L / L_{\odot}-2.85 X_{\mathrm{s}}$.

Since Hamann \& Koesterke (1998) suggested that these mass loss rates may be overestimated, we calculated evolutionary models in which the mass loss rate given by Eq. (1) is multiplied by $1 / 3$.

Also, enhanced mass loss due to rotation is included:

$\dot{M} / \dot{M}\left(v_{\text {rot }}=0\right)=1 /(1-\Omega)^{\xi}$,

where $\xi=0.43, \Omega=v_{\text {rot }} / v_{\text {crit }}$ and $v_{\text {crit }}^{2}=G M(1-\Gamma) / R$ with $\Gamma=L / L_{\mathrm{Edd}}=\kappa L /(4 \pi c G M)$ is the Eddington factor, $G$ is gravitational constant, $M$ is mass, $R$ radius, $\kappa$ opacity, $v_{\text {rot }}$ rotating velocity and $v_{\text {crit }}$ critical rotational velocity (Langer 1998). 
The transport of angular momentum in our code is formulated as a diffusive process:

$\left(\frac{\partial \omega}{\partial t}\right)_{m}=\frac{1}{i}\left(\frac{\partial}{\partial m}\right)_{t}\left[\left(4 \pi r^{2} \rho\right)^{2} i v\left(\frac{\partial \omega}{\partial m}\right)_{t}\right]_{-} \frac{2 w}{r}\left(\frac{\partial r}{\partial t}\right)_{m} \frac{1}{2} \frac{\mathrm{d} \ln i}{\mathrm{~d} \ln r}$,

where $v$ is the turbulent viscosity and $i$ is the specific angular momentum of a shell at mass coordinate $m$. Factor $(1 / 2)(\mathrm{d} \ln i / \mathrm{d} \ln r)$ vanishes if the gyration constant $k=$ $i / r^{2}$ does not depend on $r$, i.e. if all shells are moving homologously.

A parameter $f_{\mu}=0.05$ is adopted for sensitivity of the rotationally induced mixing processes to the $\mu$-gradient (Heger et al. 2000a).

As shown by Meynet \& Maeder (1997), $\mu$-gradients can efficiently suppress rotationally induced transport processes. The strenght of this inhibiting effect is described by the parameter $f_{\mu}=0 \ldots 1$ (Pinsonneault et al. 1989). A value of $f_{\mu}=0.05$ reproduces the best observations of the enrichement in the surface of the main sequence stars with products of CNO process.

At the surface of the star, the angular momentum contained in the layers which are lost due to stellar wind gets removed from the star:

$\dot{J}=\dot{M} j_{\text {spec }}$

where $j_{\text {spec }}$ is the average angular momentum at the surface of the star and $\dot{M}$ the stellar wind mass loss rate. The turbulent viscosity, $v$, is determined as the sum of the convective and semiconvective diffusion coefficients and those from rotationally induced instabilities (dynamical shear, SolbergHøiland, secular shear, and Goldreich-Schubert-Fricke instability and Eddington-Sweet circulation). Rotationally induced mixing processes and angular momentum transport through the stellar interior are described in detail by Heger et al. (2000a).

The code calculates the simultaneous evolution of two stellar components of a binary system and computes mass transfer within the Roche approximation (Kopal 1978). Mass loss from the Roche lobe filling component through the first Lagrangian point is given by Ritter (1988) as:

$\dot{M}=\dot{M}_{0} \exp \left(R-R_{1}\right) / H_{\mathrm{p}}$

with $\dot{M}_{0}=\rho v_{\mathrm{s}} Q / \sqrt{e}$, where $e$ is the base of the natural logarithm, $H_{\mathrm{p}}$ is the photospheric pressure scale height, $\rho$ is the density, $v_{\mathrm{s}}$ the velocity of sound, and $Q$ the effective cross-section of the stream through the first Lagrangian point according to Meyer \& Meyer-Hofmeister (1983). The time scales for synchronization and circularization of the binary orbit as well as spin-orbit coupling are given by Zahn (1977). The specific angular momentum of the accreted matter is determined by integrating the equation of motion of a test particle in the Roche potential (Wellstein 2001, accretion stream impacts directly on the secondary star).

We calculate the evolution of binary systems in detail until Case AB mass transfer starts. Then we remove the hydrogen rich envelope from the primary, until only $5 \%$ of the hydrogen is left in the envelope. This is the point where we assume that the primary shrinks and Case AB stops. On the other side, we calculate the secondary star assuming accretion with a mass transfer of $10^{-4} M_{\odot} \mathrm{yr}^{-1}$. We calculate the Kelvin-Helmholtz time scale of the primary on the beginning of Case AB mass transfer:

$t_{\mathrm{KH}}=2 \times 10^{7} M_{1}^{2} /\left(L_{1} R_{11}\right) \mathrm{yr}$,

where $M_{1}, L_{1}$ and $R_{11}$ are mass, luminosity and Roche radius (in Solar units) of the primary star at the onset of Case AB mass transfer. Mass transfer rate is:

$\dot{M}_{\mathrm{tr}}=\left(M_{1}-M_{\mathrm{He}}\right) / t_{\mathrm{KH}}$

where $M_{\mathrm{He}}$ is the mass of the helium core with $\sim 5 \%$ of hydrogen on the surface, i.e. the initial WR mass. We calculate the orbit change assuming constant mass transfer rate calculated by Eq. (7) and an average accretion efficiency $(\beta)$ which is the same as for the fast phase of Case A, since Case AB also happens on the thermal time scale. Matter that has not been accreted on the secondary leaves the system with a specific angular momentum which corresponds to the secondary orbital angular momentum (King et al. 2001). Stellar wind mass loss is neglected.

We follow the evolution of the binary system, again in detail, until the primary finishes carbon core burning. We assume that after this the system is disrupted by the SN explosion of the primary and we model the secondary further as a single star. Stellar wind mass loss for red supergiants is given by Nieuwenhuijzen \& de Jager (1990).

Magnetic fields generated by differential rotation are included according to Spruit (2002). The rate at which the field is amplified is determined by the differential rotation. Differentional rotation rate (rotation gradient) is a function of a radial coordinate only ("shellular rotation") and is given by:

$q=\frac{\partial \ln \Omega_{\star}}{\partial \ln r}=\frac{r \partial_{r} \Omega_{\star}}{\Omega_{\star}}$.

The dynamo process requires a minimum rotation gradient $q_{\text {min }}$ to operate.

The effective radial viscosity produced by the magnetic field is:

$v_{r e}=\frac{v_{e 0} v_{e 1}}{v_{e 0}+v_{e 1}} f(q)$,

where

$v_{e 0}=r^{2} \Omega_{\star} q^{2}\left(\frac{\Omega_{\star}}{N_{\mu}}\right)^{4}$,

$v_{e 1}=r^{2} \Omega_{\star} \max \left[\left(\frac{\Omega_{\star}}{N_{\mathrm{T}}}\right)^{1 / 2}\left(\frac{\kappa}{r^{2} N_{\mathrm{T}}}\right)^{1 / 2}, q^{2}\left(\frac{\Omega_{\star}}{N_{\mathrm{T}}}\right)^{4}\right]$,

$r$ is the radial coordinate, $\Omega_{\star}$ is the rotation rate of the star, is $N_{\mu}$ the compositional buoyancy frequency, $N_{\mathrm{T}}$ is the thermal part of buoyancy frequency, $q$ is the rotational gradient and $q_{\text {min }}$ is minimum rotational gradient necessary for the dynamo to operate:

$f(q)=1-q_{\min } / q,\left(q>q_{\min }\right)$,

and

$f(q)=0,\left(q \leq q_{\min }\right)$. 
The factor $f(q)$ causes the stress to vanish smoothly as the gradient of the rotation rate approaches the minimum value required for dynamo action.

Fluid motions involved in the dynamo process also imply a certain amount of mixing. The effective diffusivity is given as:

$D_{e}=\frac{D_{e 0} D_{e 1}}{D_{e 0}+D_{e 1}} f(q)$,

where $f(q)$ is defined by Eq. (11) and

$$
\begin{aligned}
& D_{e 0}=r^{2} \Omega_{\star} q^{4}\left(\frac{\Omega_{\star}}{N_{\mu}}\right)^{6}, \\
& D_{e 1}=r^{2} \Omega_{\star} \max \left[\left(\frac{\Omega_{\star}}{N_{\mathrm{T}}}\right)^{3 / 4}\left(\frac{\kappa}{r^{2} N_{\mathrm{T}}}\right)^{3 / 4}, q^{2}\left(\frac{\Omega_{\star}}{N_{\mathrm{T}}}\right)^{6}\right] .
\end{aligned}
$$

\section{Models without magnetic field}

\subsection{Single stars}

It was shown by Meynet \& Maeder (1997) and Heger et al. (2000a) that transport of angular momentum in stellar interiors depends strongly on the efficiency of the inhibition of the rotationally induced mixing processes by $\mu$-gradient. When rotationally induced mixing processes are not inhibited by $\mu$-gradient, stellar rotation remains close to rigid rotation. The star loses matter from the surface which carries away angular momentum. Layers beneath the lost ones expand, and due to local angular momentum conservation, spin down. The star reestablishes rigid rotation by transporting angular momentum from the core to the surface. When the rotationally induced mixing processes are inhibited by the $\mu$-gradient, angular momentum cannot be efficiently transported between the core and the envelope. The result is differential rotation between these two stellar regions. In this paper we present models which include the inhibiting effect of $\mu$-gradient on rotationally induced mixing processes.

We calculate the evolution of a $20 M_{\odot}$ star until the end of core carbon burning. This star is a rigidly rotating star on the ZAMS (Fig. 1, three dots-dashed line) with an initial surface velocity of $v_{\text {surf }}=200 \mathrm{~km} \mathrm{~s}^{-1}$, which is a typical value for these stars (Fukuda 1982). During its hydrogen core burning phase, this star loses mass due to a stellar winds $\left(\sim 10^{-8} M_{\odot} \mathrm{yr}^{-1}\right)$. Matter lost from the stellar surface carries away angular momentum, the surface layers spin down, but there is no efficient transport of angular momentum between the core and the envelope, so the core does not spin down significantly. At the time of helium ignition (Fig. 1, dot-dashed line), the specific angular momentum of the core is $j \sim 10^{17} \mathrm{~cm}^{2} \mathrm{~s}^{-1}$ at $3 M_{\odot}$ (further in the paper, specific angular momentum values are always given at $3 M_{\odot}$, since that is the mass of the final core before supernova explosion). After core hydrogen exhaustion, the star evolves into a red supergiant, its core contracts while the envelope expands. This leads to a spin-up of the core and a spin-down of the envelope. The envelope is convective and rotating almost rigidly with a very low rotational velocity $\left(\sim 0.1 \mathrm{~km} \mathrm{~s}^{-1}\right)$. The core is rigidly rotating with a maximum rotational velocity of $\sim 130 \mathrm{~km} \mathrm{~s}^{-1}$. The core and the envelope are separated by layers that have a large $\mu$-gradient. This suppresses rotationally

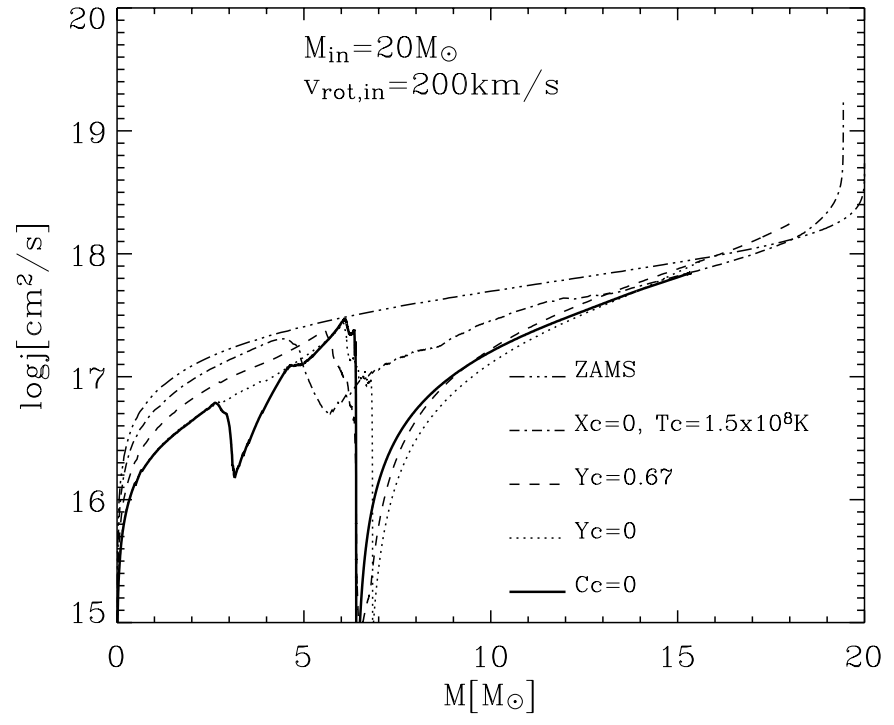

Fig. 1. Specific angular momentum profiles of a $20 M_{\odot}$ single star on the hydrogen ZAMS (three dots-dashed line), when helium ignites in the core, (dot-dashed line), when the central helium abundance is $67 \%$ (dashed-line), in the moment of core helium exhaustion (dotted line) and at core carbon exhaustion (solid line).

induced mixing and angular momentum is not efficiently transported, so that the core is not slowed down by the slow rotation of the envelope. At the end of helium core burning the specific angular momentum of the core is $j \sim 5 \times 10^{16} \mathrm{~cm}^{2} \mathrm{~s}^{-1}$ (Fig. 1, dotted line). During further evolution, i.e., core carbon burning, the stellar core does not lose any significant amounts of angular momentum.

To investigate the behaviour of higher mass stars, we modeled the evolution of a $42 M_{\odot}$ star with the same initial surface rotational velocity $v_{\text {surf }}=200 \mathrm{~km} \mathrm{~s}^{-1}$. The star is a rigidly rotating star on the ZAMS with the specific angular momentum profile shown in Fig. 3 (dot-dashed line). As we already explained for the previous example, the star loses mass due to a stellar wind $\left(\sim 10^{-7} M_{\odot} \mathrm{yr}^{-1}\right)$. Since the stellar wind mass loss rate is one order of magnitude higher than for a $20 M_{\odot}$ star, the $42 M_{\odot}$ star loses more matter and angular momentum during the core hydrogen burning phase. When helium ignites in the core, the specific angular momentum at $3 M_{\odot}$ is $\sim 8 \times 10^{16} \mathrm{~cm}^{2} \mathrm{~s}^{-1}$. The star becomes a $\sim 38 M_{\odot}$ red supergiant with central helium burning in a convective core of $\sim 12 M_{\odot}$ and a convective envelope of $\sim 20 M_{\odot}$. Due to stellar wind mass loss of $\sim 10^{-4} M_{\odot} \mathrm{yr}^{-1}$, the mass of the envelope rapidly decreases and reaches $\sim 10 M_{\odot}$ at the end of our calculations.

We follow the evolution of this star until it burned $~ 30 \%$ of the helium in its core. The specific angular momentum of the core (at $3 M_{\odot}$ ) at that moment is $\sim 6.5 \times 10^{16} \mathrm{~cm}^{2} \mathrm{~s}^{-1}$. If we assume that the angular momentum of the core decreases further during helium core burning with the same rate, the specific angular momentum of the core at the moment of helium exhaustion would be $\sim 3.5 \times 10^{16} \mathrm{~cm}^{2} \mathrm{~s}^{-1}$. We see in Fig. 1 that there is no angular momentum loss from the core during core carbon burning, so we can conclude that this star might produce a collapsar and in case that the hydrogen envelope is lost during the red the supergiant phase, a gamma-ray burst can be the result. 


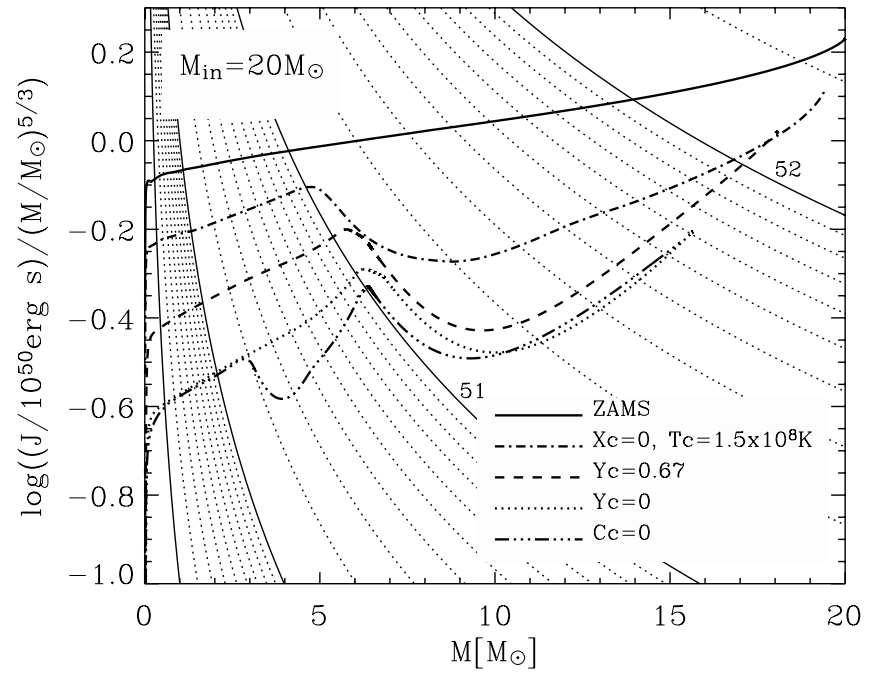

Fig. 2. Logarithm of the integrated angular momentum, $J(m)=$ $\int_{0}^{M} j\left(m^{\prime}\right) \mathrm{d} m^{\prime}$ divided by $m^{5 / 3}$, as a function of the mass coordinate, $m$, for a $20 M_{\odot}$ star for the same evolutionary stages as shown in Fig. 1 . The thin lines give a logarithmic scale of levels of constant $J$ labeled with $\log (J /(\mathrm{erg} \mathrm{s}))$.

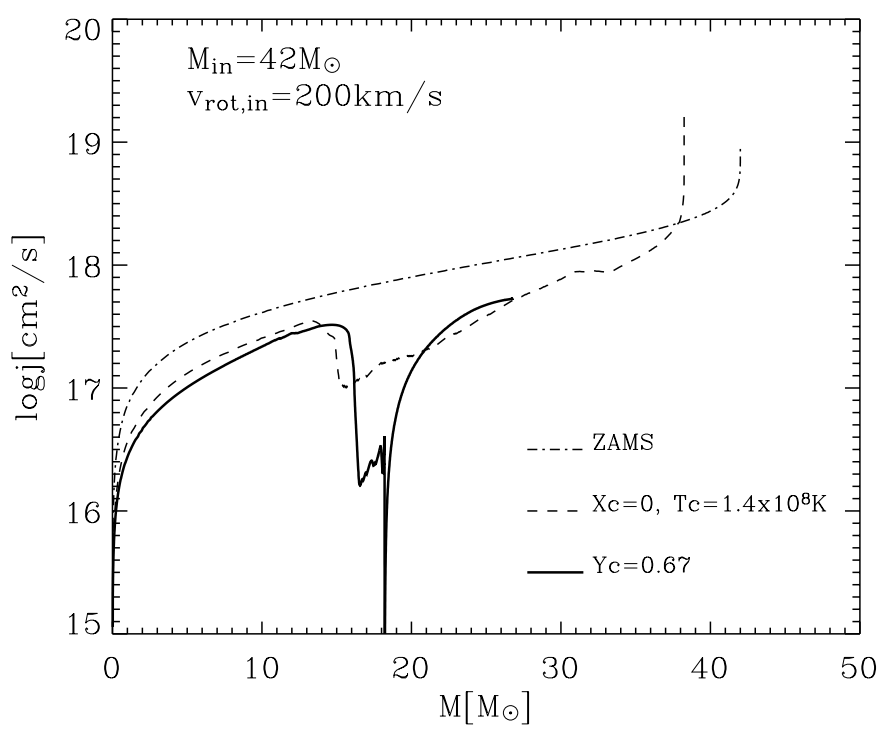

Fig. 3. Specific angular momentum profiles of a $42 M_{\odot}$ single star on the hydrogen ZAMS (dot-dashed line), when helium ignites in the center (dotted line), and when the central helium abundance is $67 \%$ (solid line).

For a rigidly rotating body of constant density $\rho_{0}$, the total angular momentum, $J(m)$, enclosed below the mass coordinate, $m$, is $J(m)=\int_{0}^{M} j\left(m^{\prime}\right) \mathrm{d} m$. In Figs. 2 and 4 we plot the logarithm of $J(m) / m^{5 / 3}$ as a function of mass and a grid of lines of $J=$ const. If angular momentum is transported throughout the surface defined by a mass coordinate $m, J(m)$ drops. If no such transport occurs, $J(m)$ remains constant. If a line of constant $J$ is followed from one evolutionary stage to another, it can be seen to what mass coordinate the angular momentum has been transported in the star during the time between the two evolutionary stages.

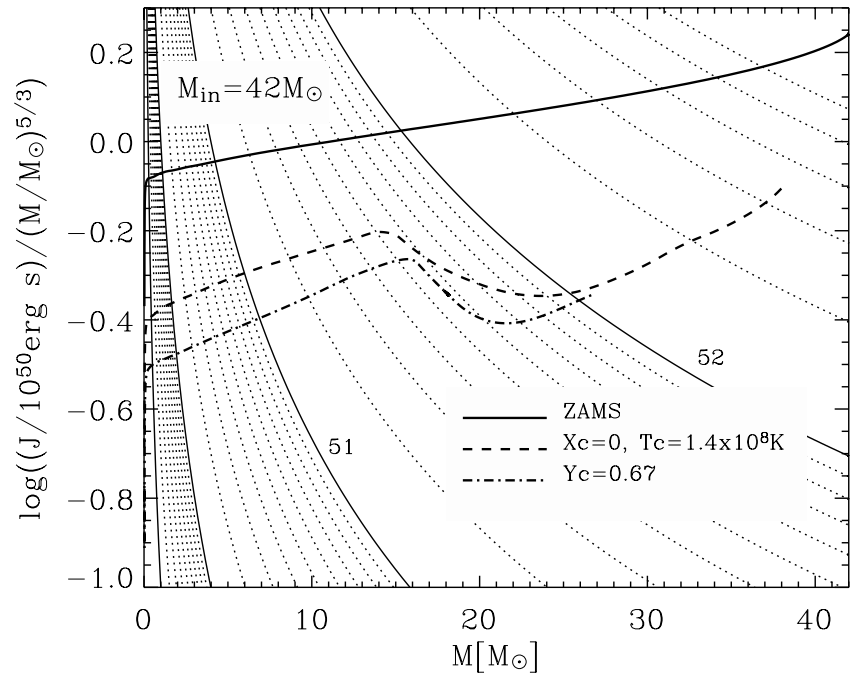

Fig. 4. Logarithm of the integrated angular momentum $J(m)=$ $\int_{0}^{M} j\left(m^{\prime}\right) \mathrm{d} m^{\prime}$ divided by $m^{5 / 3}$, as a function of the mass coordinate $m$ for a $42 M_{\odot}$ star for the same evolutionary stages as shown in Fig. 3. The thin lines give a logarithmic scale of levels of constant $J$ labeled with $\log (J /($ erg s $))$.

\subsection{Binary systems}

A star evolving in a binary system and accreting matter from the companion, increases its surface angular momentum. If this angular momentum can be transported efficiently through the stellar interior, the star may evolve into a red supergiant that has a rapidly spinning core with sufficient specific angular momentum to produce a collapsar.

To check if accretion can add enough angular momentum to the core, we modeled the evolution of a rotating binary system with initial masses of $M_{1, \text { in }}=56 M_{\odot}$ and $M_{2, \text { in }}=33 M_{\odot}$ and an initial orbital period of $p_{\text {in }}=6$ days. The binary system quickly synchronizes during the main sequence evolution. Due to this synchronization, both stars lose angular momentum and their initial surface rotational velocities are $92 \mathrm{~km} \mathrm{~s}^{-1}$ for the primary and $64 \mathrm{~km} \mathrm{~s}^{-1}$ for the secondary which is much slower than the typical values for single stars of these masses (Heger et al. 2000a). This means that stars in binary systems lose a significant amount of angular momentum due to synchronization. The angular momentum loss increases with the initial orbital period.

Evolutionary tracks of the primary and the secondary star are given in Figs. 5 and 6. The primary is initially the more massive star; it evolves faster and fills its Roche lobe during hydrogen core burning. The binary system enters Case A of mass transfer (dotted line, Fig. 5). The primary loses matter with a high mass transfer rate $\left(\dot{M}_{\mathrm{tr}}^{\max } \sim 3.2 \times 10^{-3} M_{\odot} \mathrm{yr}^{-1}\right)$ and decreases in luminosity. During the fast phase of Case A mass transfer, the primary loses $\sim 19 M_{\odot}$ and the secondary accretes only about $15 \%$ of that matter due to the stellar wind mass loss caused by rotation close to break-up (Petrovic et al. 2004). After the fast process of Case A mass transfer, the primary continues to expand on a nuclear time scale and transfer mass onto the secondary star with mass transfer rate of $\sim 10^{-6} M_{\odot} \mathrm{yr}^{-1}$ (slow phase of Case A). At the end of core 


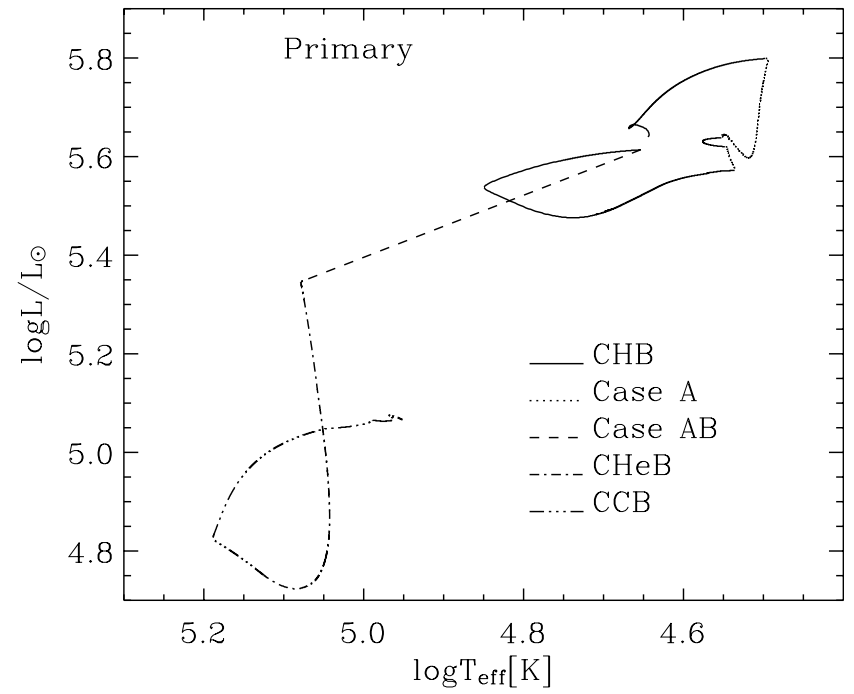

Fig. 5. Evolutionary track of the primary in HR diagram. Solid line: core hydrogen burning phase before and after Case A mass transfer. Dotted line: Case A mass transfer. Dashed line: connection between last calculated model at the onset of Case AB and first calculated model when the primary is a hydrogen-free WR star. Dash-dotted line: core helium burning phase. Dot-dashed line: core carbon burning phase.

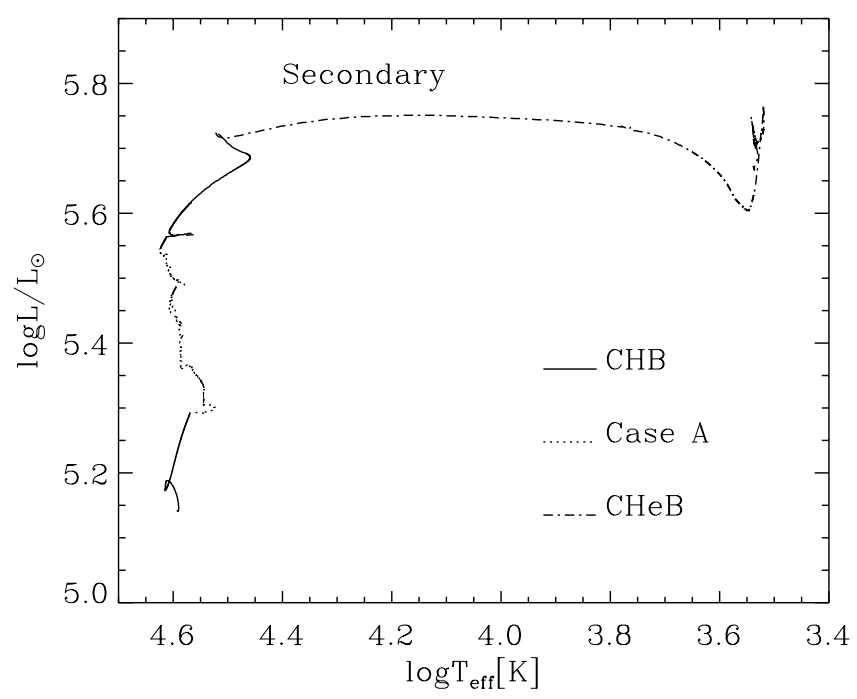

Fig. 6. Evolutionary track of the secondary in the HR diagram. Solid line: core hydrogen burning phase before and after Case A and Case AB mass transfer. Dotted line: Case A mass transfer. Dash-dotted line: core helium burning phase.

hydrogen burning the primary contracts and thus Roche lobe overflow (RLOF) stops. When the primary starts hydrogen shell burning $\left(M=16.6 M_{\odot}\right)$ it expands again, fills its Roche lobe and Case $\mathrm{AB}$ mass transfer starts. We assume that this mass transfer stops when the WR star has $5 \%$ of hydrogen on its surface $\left(M_{\mathrm{WR}}\left(X_{\mathrm{s}}=0.05\right)=14.8 M_{\odot}\right)$. More details about the evolution of this system up to $\mathrm{WR}+\mathrm{O}$ phase can be seen in Petrovic et al. (2004). As explained in Sect. 2, we continue following the evolution of the binary system with a hydrogenfree WR star. The dashed line in Fig. 5 connects the last calculated model at the onset of Case $\mathrm{AB}$ and the first calculated

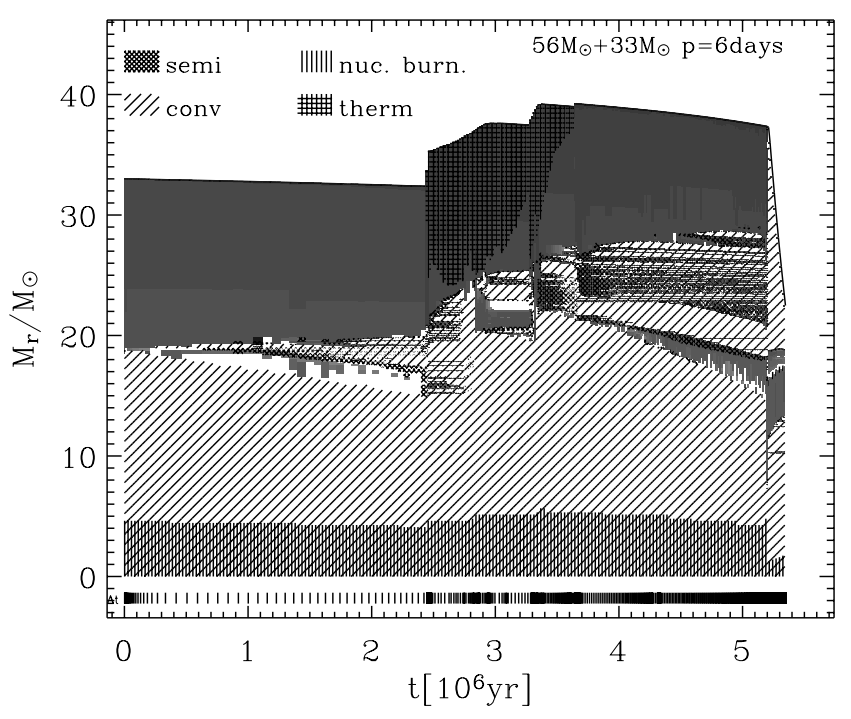

Fig. 7. Evolution of the internal structure of a rotating $33 M_{\odot}$ secondary from the ZAMS until red supergiant phase $Y_{\mathrm{c}}=0.67$. Convection is indicated with diagonal hatching, semiconvection with crossed hatching and thermohaline mixing with straight crossed hatching. The hatched area at the bottom indicates nuclear burning. Gray shaded areas represent regions with rotationally induced mixing (intensity is indicated with different shades, the darker the colour, the stronger rotational mixing). The topmost solid line corresponds to the surface of the star.

model when the primary is a hydrogen-free WR star. The initial hydrogen-free WR star is $M_{\mathrm{WR}}=12.8 M_{\odot}$ with an effective temperature of $\sim 1.2 \times 10^{5} \mathrm{~K}$, and it loses $\sim 8 M_{\odot}$ due to WR mass loss during core helium burning. Due to this mass loss, the luminosity of the primary decreases (dash-dotted line Fig. 5). The primary ends carbon core burning (dot-dashed line Fig. 5) as $\sim 4.5$ solar mass star. The orbital period of the binary system is $\sim 13.5$ days when the primary explodes in a supernova.

The secondary begins its evolution as a $33 M_{\odot}$ core hydrogen burning star. It accretes $\sim 3 M_{\odot}$ during the fast phase and $\sim 4 M_{\odot}$ during the slow phase of Case A mass transfer. Due to this mass gain, the luminosity of the secondary increases (Fig. 6). As we already mentioned, the primary loses $\sim 1.8 M_{\odot}$ during Case $\mathrm{AB}$. We assume the same average accretion efficiency during this mass transfer as during the fast phase of Case A mass transfer $(\beta=0.15)$, since they both take place on the thermal time scale. This means that the secondary accretes $\sim 0.25 M_{\odot}$ and after Case $\mathrm{AB}$ mass transfer, it is still a core hydrogen burning star, more massive than at the beginning of its main sequence evolution $\left(39.25 M_{\odot}\right)$. After the secondary exhausted all hydrogen in the core, it contracts and increase its luminosity and effective temperature. Due to the increase of temperature in the envelope, the star ignites hydrogen in a shell, expands and cools down drastically (dash-dotted line Fig. 6, $\left.R \approx 2300 R_{\odot}, T_{\text {eff }} \approx 3500 \mathrm{~K}\right)$. Meanwhile, the core contracts further and the core temperature increases. Helium core burning starts when $T_{\mathrm{c}} \approx 1.4 \times 10^{8} \mathrm{~K}$. We stopped detailed modeling when the secondary has $\sim 67 \%$ of helium left in the core.

Figure 7 shows the evolution of the internal structure of the secondary from the ZAMS, through Case A and Case AB mass transfer and the red supergiant phase until $Y_{\mathrm{c}}=0.67$. The 


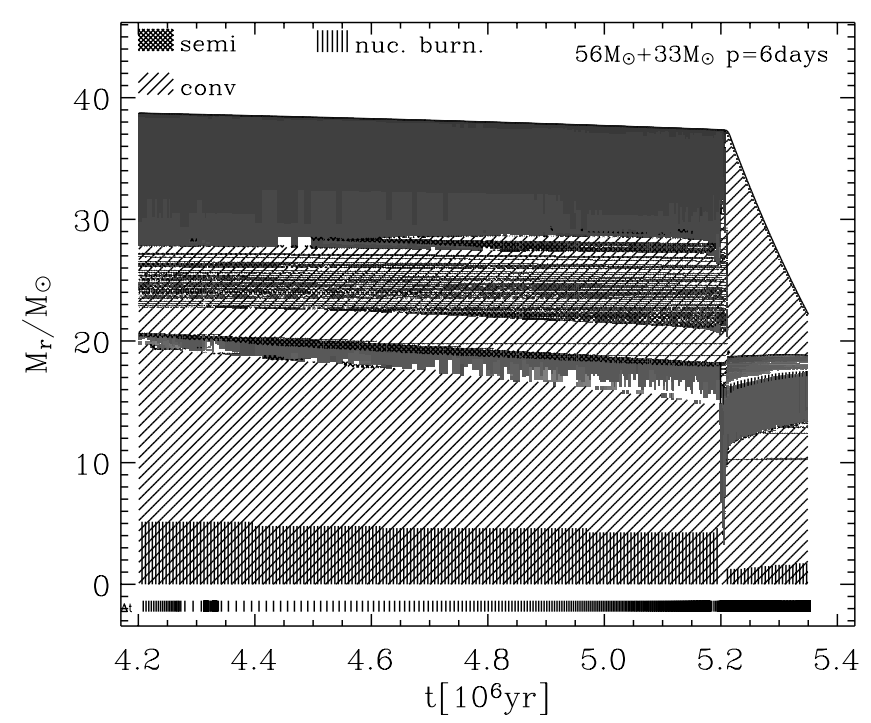

Fig. 8. Evolution of the internal structure of a rotating $33 M_{\odot}$ secondary from $\mathrm{SN}$ explosion of the primary and disruption of the system. See Fig. 7 for an explanation of the different hatching types.

secondary starts its main sequence evolution as a $33 M_{\odot}$ star with a convective core of $\sim 20 M_{\odot}$. Rotationally induced mixing processes take place in the radiative envelope of the star. The secondary then accretes $\sim 3 M_{\odot}$ during the fast phase and $\sim 4 M_{\odot}$ during the slow phase of Case A mass transfer. Heavier elements accreted on the surface are relocated by thermohaline mixing process and the convective core increases its mass $\left(\sim 25 M_{\odot}\right)$. The secondary becomes a rejuvenated $\sim 39 M_{\odot}$ core hydrogen burning star in a WR+O binary system. The system is likely to be disrupted by the explosion of the primary while the secondary is still a main sequence star. Details of the evolution of the internal structure of the secondary after the primary exploded are shown in Fig. 8. After hydrogen is exhausted in the core, the secondary becomes a $\sim 37 M_{\odot}$ red supergiant with central helium burning in convective core of $\sim 12 M_{\odot}$ and a convective envelope of $\sim 20 M_{\odot}$. Due to stellar wind mass loss of $\sim 10^{-4} M_{\odot} \mathrm{yr}^{-1}$, the mass of the envelope rapidly decreases and reaches $\sim 10 M_{\odot}$ at the end of our calculations.

The modeled binary system starts its evolution with both components synchronized to the orbital rotation. The surface rotational velocity of the secondary star is $\sim 64 \mathrm{~km} \mathrm{~s}^{-1}$. When the fast Case A mass transfer starts, the secondary accretes matter from the primary with high mass transfer rates $\left(\sim 10^{-3} M_{\odot} \mathrm{yr}^{-1}\right)$. This matter carries angular momentum and spins up the top layers of the secondary star (Fig. 9). The mass transfer rate during the slow phase of Case A is significantly lower $\left(10^{-6} M_{\odot} \mathrm{yr}^{-1}\right)$ and the surface rotational velocity of the secondary increases to about $200 \mathrm{~km} \mathrm{~s}^{-1}$. For Case AB we assumed a mass transfer rate of $\sim 10^{-4} M_{\odot} \mathrm{yr}^{-1}$ (Sect. 2) and this spins up the surface of the secondary to $\sim 500 \mathrm{~km} \mathrm{~s}^{-1}$. When the secondary spins up to close to critical rotation it loses more mass according to Eq. (2). High mass loss decreases the net accretion efficiency and also removes angular momentum from the secondary star. The secondary star is also spundown by tidal forces that tend to synchronize it with the orbital motion (Petrovic et al. 2004). After Case AB mass transfer,

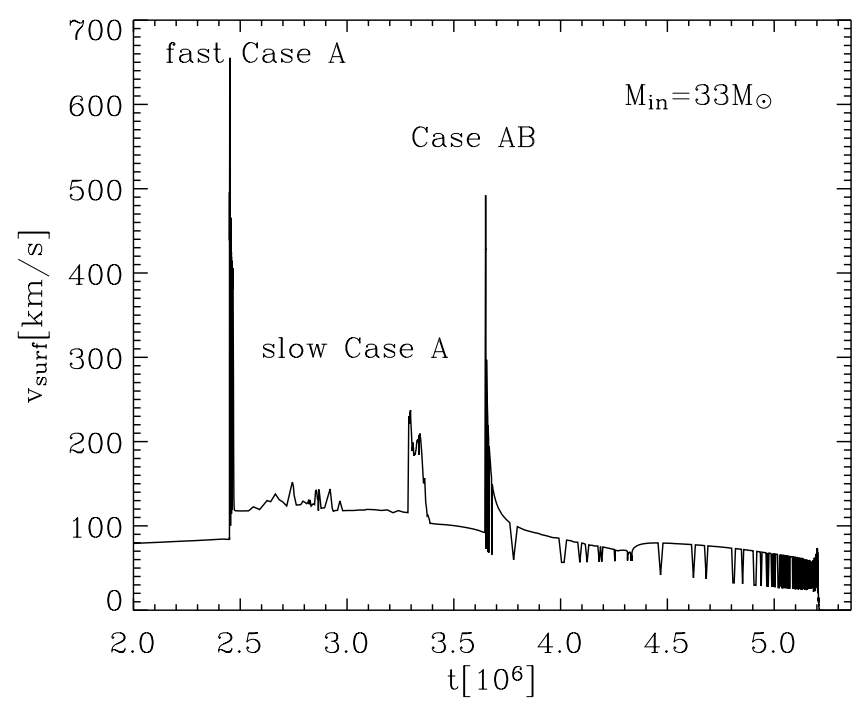

Fig. 9. Surface rotational velocity of the secondary star. Matter transfered from the primary with high mass transfer rate $\sim 10^{-3}$ $10^{-4} M_{\odot} \mathrm{yr}^{-1}$ during the fast phase of Case $\mathrm{A}$ and Case $\mathrm{AB}$ spins up the surface layers of the secondary up to $500-700 \mathrm{~km} \mathrm{~s}^{-1}$. During the slow phase of Case A mass transfer rate is lower $\left(\sim 10^{-6} M_{\odot} \mathrm{yr}^{-1}\right)$ and surface rotational velocity increases up to $\sim 200 \mathrm{~km} \mathrm{~s}^{-1}$. After Case AB mass transfer the secondary synchronizes with the orbital motion in the WR+O binary system. After the SN explosion of the primary, the secondary star evolves into a red supergiant with slowly rotating envelope of $\sim 0.02 \mathrm{~km} \mathrm{~s}^{-1}$.

the secondary will synchronize with the orbital motion in the $\mathrm{WR}+\mathrm{O}$ binary system. After the $\mathrm{SN}$ explosion of the primary, the secondary becomes a red supergiant with a very slowly rotating envelope and its surface rotational velocity drops to less than $0.02 \mathrm{~km} \mathrm{~s}^{-1}$.

Figure 10 shows rotational velocity profiles of the secondary star in different evolutionary phases. The surface of the secondary gains additional angular momentum during mass transfer, as we already explained. This angular momentum is transported through the stellar interior, and into the core. The result is that the stellar core spins faster on the helium ZAMS (Fig. 10, three dots-dashed line) than at the beginning of hydrogen core burning (Fig. 10, solid line). After this, the core contracts and further increases its rotational velocity, and the envelope expands and slows down. When the central abundance of helium is around $67 \%$, the maximum rotational velocity of the core is $\sim 100 \mathrm{~km} \mathrm{~s}^{-1}$.

Figure 11 shows specific angular momentum profiles of the secondary at different points of its evolution. The specific angular momentum of the secondary increases significantly due to fast Case A mass transfer (Fig. 11, dotted line). After this, the secondary loses angular momentum due to stellar wind mass loss, but also gains certain amount through slow Case A and Case AB mass transfer (Fig. 11, dashed and dot-dashed line). The result is that the core has a larger specific angular momentum when central helium burning starts than at the beginning of hydrogen core burning. After core hydrogen exhaustion, the secondary evolves into a red supergiant, the core contracts and the envelope expands. This leads to a spin-up of the core and a spin-down of the envelope. The specific angular 


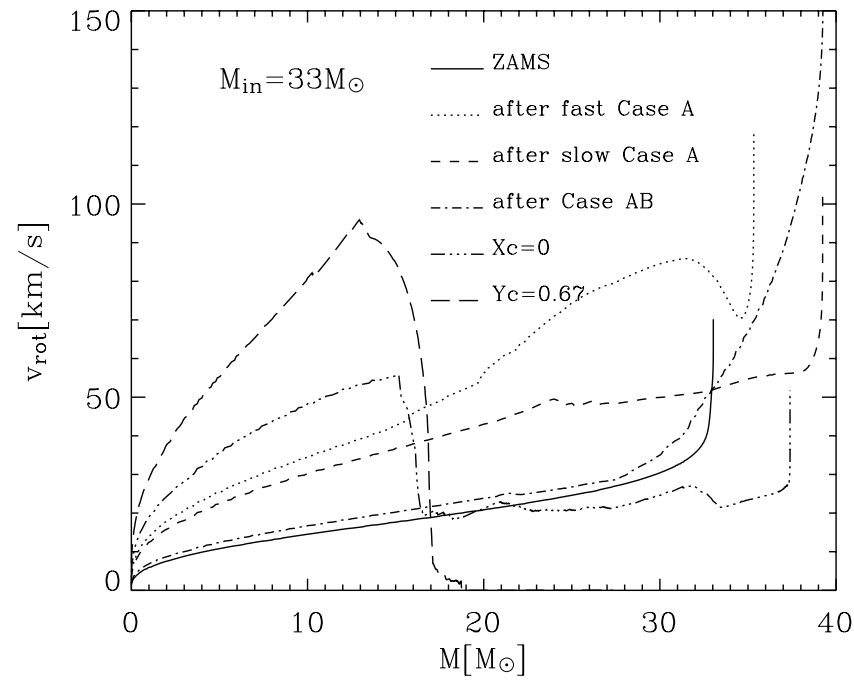

Fig. 10. Rotational velocity profiles of the secondary star on the hydrogen ZAMS (solid line), after the fast (dotted line) and the slow (short dashed line) phase of Case A mass transfer, after Case AB mass transfer (dash-dotted line), when helium ignites in the core (three dotsdashed line) and when the central helium abundance is $67 \%$ (long dashed-line).

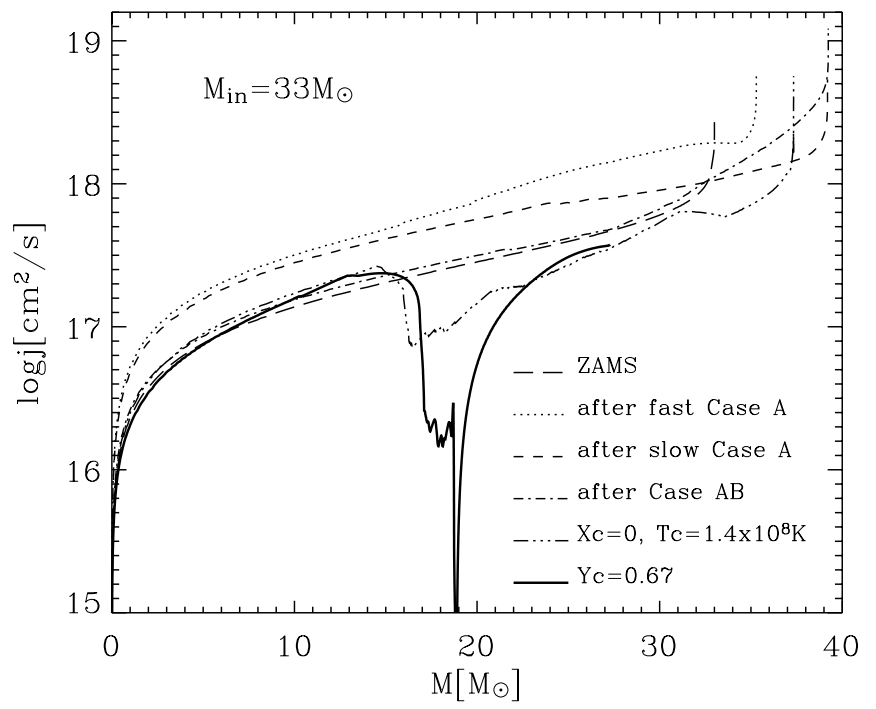

Fig. 11. Specific angular momentum profiles of the secondary star on the hydrogen ZAMS (long dashed line), after fast (dotted line) and slow (short dashed line) Case A mass transfer, after Case AB mass transfer (dash-dotted line), when helium ignites in the core (three dots-dashed line) and when the central helium abundance is $67 \%$ (solid line).

momentum of the core at $3 M_{\odot}$ is $\sim 5.5 \times 10^{16} \mathrm{~cm}^{2} \mathrm{~s}^{-1}$ (Fig. 11, three dot-dashed line). The envelope is convective and slowly rotating $\left(\sim 0.02 \mathrm{~km} \mathrm{~s}^{-1}\right)$. The core is rigidly rotating with maximum rotational velocity of $\sim 100 \mathrm{~km} \mathrm{~s}^{-1}$. The core and the envelope are separated by layers that have a high $\mu$-gradient. Angular momentum is not efficiently transported through these layers, so the core is not slowed down by the slow rotation of the envelope. When a third of the central helium supply is exhausted, the core (at $3 M_{\odot}$ ) has a specific angular momentum of $\sim 5 \times 10^{16} \mathrm{~cm}^{2} \mathrm{~s}^{-1}$. If we assume that the angular momentum of

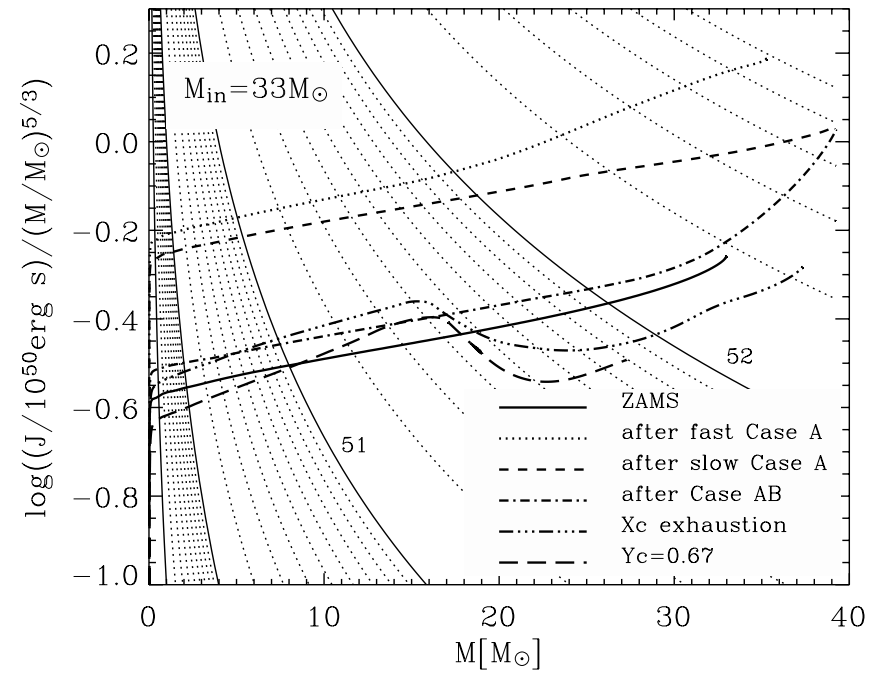

Fig. 12. Logarithm of the integrated angular momentum $J(m)=\int_{0}^{M} j\left(m^{\prime}\right) \mathrm{d} m^{\prime}$ divided by $m^{5 / 3}$, as a function of the mass coordinate $m$ for a $33 M_{\odot}$ secondary star for the same evolutionary stages as shown in Fig. 11. The thin lines give a logarithmic scale of levels of constant $J$ labeled with $\log (J /$ (erg s)).

the core decreases further during helium core burning with the same rate, specific angular momentum of the core at the moment of helium exhaustion is expected to be $\sim 4 \times 10^{16} \mathrm{~cm}^{2} \mathrm{~s}^{-1}$. As we have already seen for single stars, there is no angular momentum loss from the core during core carbon burning, so we can conclude that this star has enough angular momentum to produce a collapsar and, in the case that the hydrogen envelope is lost during red supergiant phase, a gamma-ray burst.

The gain of angular momentum of an accreting star is proportional to the amount of accreted matter. We show in Fig. 13 specific angular momentum profiles of the secondary $\left(X_{\mathrm{c}}=0\right.$, $T_{\mathrm{c}}=1.4 \times 10^{8} \mathrm{~K}$ ) assuming the accretion of $0.25,1.4$ and $5 M_{\odot}$ during Case AB mass transfer. We see that the specific angular momentum of the core is higher if the star accreted more matter during mass transfer. The specific angular momentum of the core at $3 M_{\odot}$ increases from $6 \times 10^{16} \mathrm{~cm}^{2} \mathrm{~s}^{-1}$ to $8 \times 10^{16} \mathrm{~cm}^{2} \mathrm{~s}^{-1}$.

\section{Models with magnetic field}

\subsection{Single stars}

We model the evolution of a single $42 M_{\odot}$ star with an initial rotational surface velocity of $200 \mathrm{~km} \mathrm{~s}^{-1}$ and magnetic field included. Figure 14 shows specific angular momentum profiles in different phases of the evolution. We see that the initial (ZAMS) profiles of the star without magnetic field (Fig. 3, dot-dashed line) and the star with magnetic field (Fig. 14, dotdashed line) are identical and represent solid body rotation. We notice, however, that the magnetic star loses more angular momentum from the core during the main sequence evolution as well as between hydrogen core exhaustion (Fig. 14, dashed line) and core helium ignition (Fig. 14, solid line).

The star loses mass by a stellar wind. This removes angular momentum from the surface layers and slows them down. 


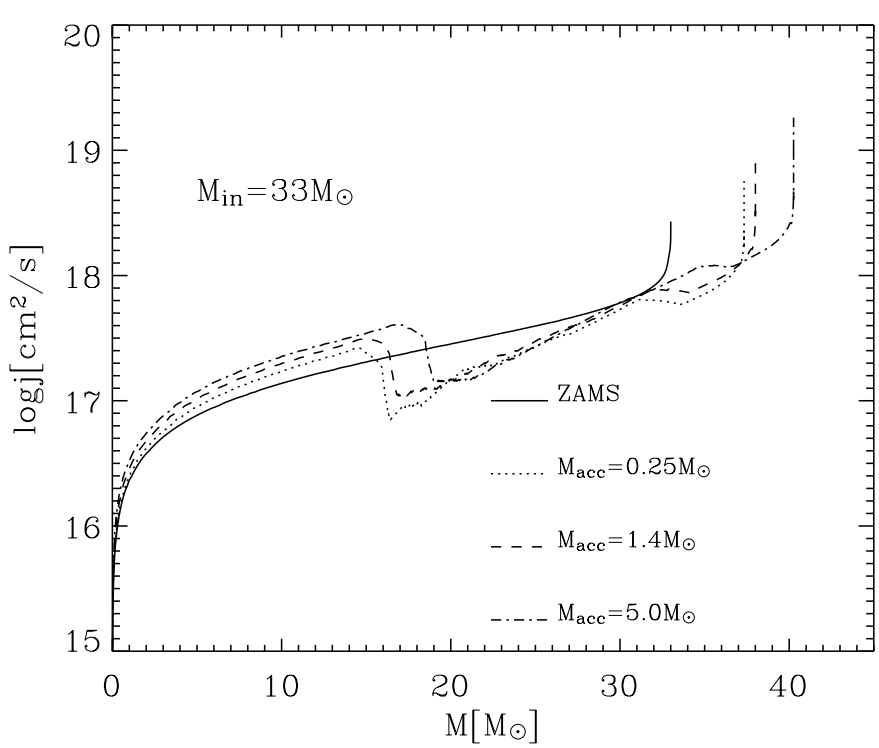

Fig. 13. Specific angular momentum profiles of the secondary star on the hydrogen ZAMS (solid line) and when helium ignites in the core $\left(T_{\mathrm{c}}=1.4 \times 10^{8} \mathrm{~K}\right)$, assuming the accretion of $0.25 M_{\odot}$ (dotted line) $1.4 M_{\odot}$ (dashed line) or $5 M_{\odot}$ (dot-dashed line) during Case AB mass transfer.

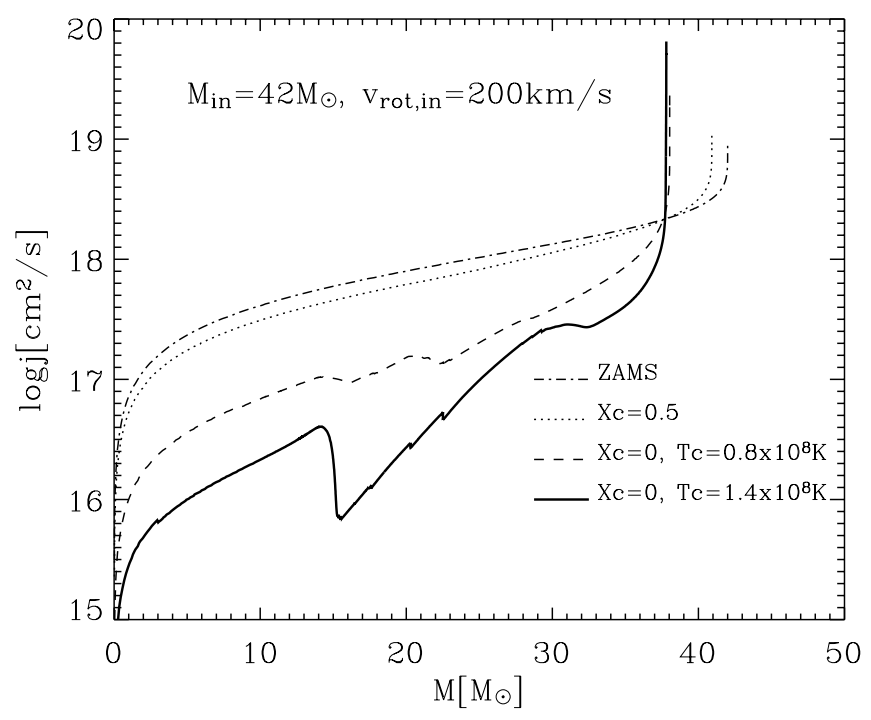

Fig. 14. Specific angular momentum profiles of the $42 M_{\odot}$ single star with magnetic fields on the hydrogen ZAMS (dot-dashed line), when $50 \%$ of hydrogen is left in the center (dotted line), when hydrogen is exhausted in the core (dashed line) and when central helium burning starts (solid line).

Since the magnetic torque keeps the star close to solid body rotation during the main sequence evolution (Fig. 15), however, angular momentum is transported from the stellar interior towards the surface. The viscosity due to the magnetic field is a few orders of magnitude larger than the one for rotational mixing $\left(\sim 10^{10-12} \mathrm{~cm}^{2} \mathrm{~s}^{-1}\right.$ compared with $\left.\sim 10^{7-8} \mathrm{~cm}^{2} \mathrm{~s}^{-1}\right)$, and it can overcome the $\mu$-gradient barrier. Therefore, the surface of the star is spun up and the core is slowed down. The surface layers rotate faster than in the corresponding non-magnetic star, and the stellar wind mass loss is higher (Fig. 16).

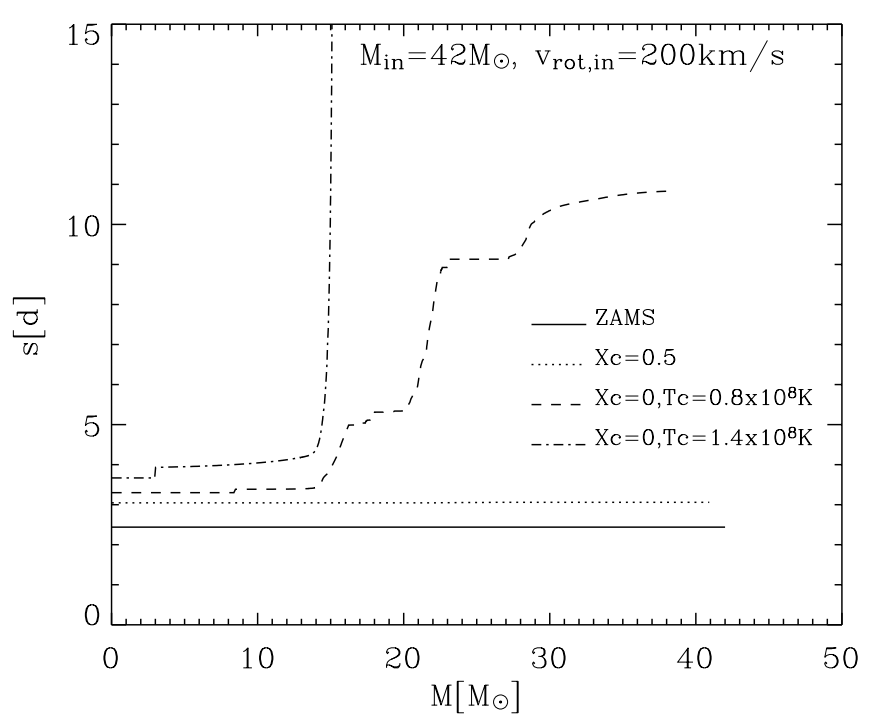

Fig. 15. Spin period profiles of the $42 M_{\odot}$ single star on the ZAMS (solid line), when $50 \%$ hydrogen is left in the center (dotted line), when hydrogen is exhausted in the core (dashed line), and when central helium burning starts (dot-dashed line).

Summarizing, the core of a magnetic star loses significantly more angular momentum during the main sequence evolution than when magnetic fields are not included. One reason is that the $\mu$-gradient does not represent a strong barrier for angular momentum transport. And second, because the surface layers are spun-up, the stellar wind mass loss is larger and this requires more angular momentum from the core to keep solid body rotation of the star. When hydrogen burning is finished $\left(T_{\mathrm{c}}=0.8 \times 10^{8} \mathrm{~K}\right)$, the specific angular momentum of the core (at $3 M_{\odot}$ ) is less than $3 \times 10^{16} \mathrm{~cm}^{2} \mathrm{~s}^{-1}$ (Fig. 14, dashed line).

When the star expands after core hydrogen burning, magnetic torques still work toward enforcing solid body rotation. The envelope expansion, however, is too fast and angular momentum transport is not efficient enough, to keep the envelope synchronized with the core (Fig. 15). Since magnetic viscosity can overcome the $\mu$-gradient, the core is slowed down and the envelope is spun up. Stellar wind mass loss is enhanced due to faster rotation and is significantly higher than in the case of the non-magnetic star (Fig. 16). The star loses significant an amount of angular momentum between hydrogen exhaustion and helium ignition and the specific angular momentum of the core (at $3 M_{\odot}$ ) at the time helium core burning starts is only $5 \times 10^{15} \mathrm{~cm}^{2} \mathrm{~s}^{-1}$

\subsection{Binary systems}

We modeled the evolution of a rotating binary system with magnetic fields, initial masses $M_{1 \text {,in }}=56 M_{\odot}$ and $M_{2, \text { in }}=$ $33 M_{\odot}$, and an initial orbital period of $p_{\text {in }}=6$ days. The binary system starts synchronized on the ZAMS with initial surface rotational velocities of $\sim 90 \mathrm{~km} \mathrm{~s}^{-1}$ for the primary and $\sim 60 \mathrm{~km} \mathrm{~s}^{-1}$ for the secondary which is, as we already mentioned for non-magnetic models, much slower than the typical values for single stars of these masses. 


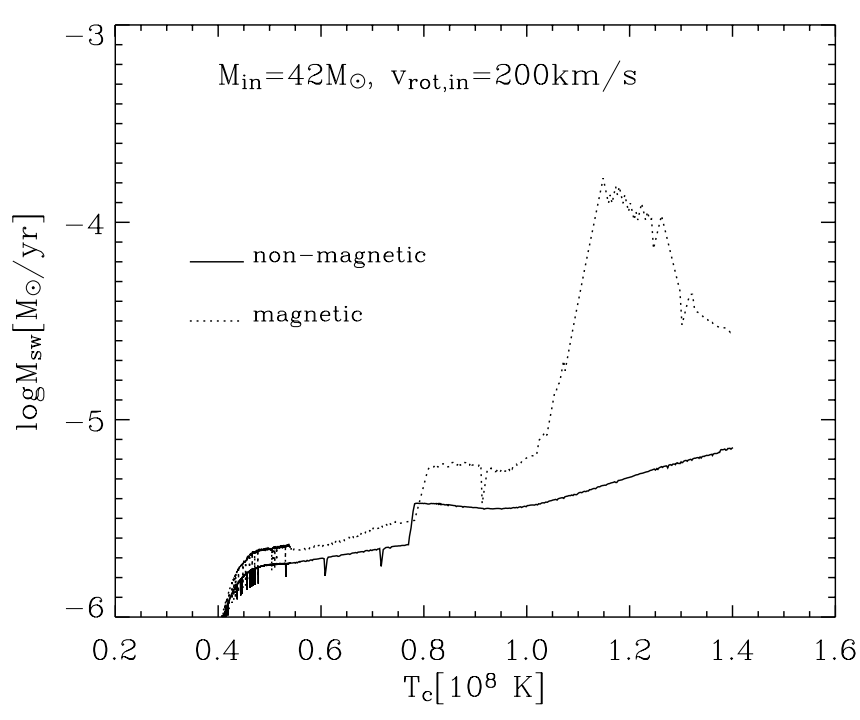

Fig. 16. Stellar wind mass loss of the magnetic (dotted line) and nonmagnetic (solid line) $42 M_{\odot}$ star. The star with magnetic fields loses more mass because magnetic torques transport angular momentum, which spins-up the surface layers and enhances mass loss.

During the fast phase of Case A mass transfer $\left(\dot{M}_{\mathrm{tr}} \sim\right.$ $\left.10^{-3} M_{\odot} \mathrm{yr}^{-1}\right)$, the primary loses $\sim 20 M_{\odot}$ and the secondary accretes only about $4.5 M_{\odot}$ of that matter due to the mass loss caused by rotation close to break-up. After the fast process of Case A mass transfer, the primary continues to expand on a nuclear time scale and to transfer mass onto the secondary star, but with much lower mass transfer rates (slow Case A, $\dot{M}_{\mathrm{tr}} \sim 10^{-6} M_{\odot} \mathrm{yr}^{-1}$ ). At the end of core hydrogen burning the primary contracts and thus RLOF stops.

Accretion increases rotational velocity of the surface of the secondary star to almost $400 \mathrm{~km} \mathrm{~s}^{-1}$ during the fast phase of Case A mass transfer. The mass transfer rate during slow Case A mass transfer is significantly lower $\left(10^{-6} M_{\odot} \mathrm{yr}^{-1}\right)$, and the surface rotational velocity of the secondary increases to about $200 \mathrm{~km} \mathrm{~s}^{-1}$.

Figure 20 shows specific angular momentum profiles of the secondary at different phases of evolution. The specific angular momentum of the secondary increases significantly due to the fast Case A mass transfer (Fig. 20, dotted line). Angular momentum is transported more efficiently through the stellar interior compared to the non-magnetic model, since the incurred magnetic torques are a few orders of magnitude more efficient in angular momentum transport than the rotational instabilities (Fig. 17). Comparing the specific angular momentum of the non-magnetic (Fig. 11) and magnetic model (Fig. 20), we notice that during fast Case $\mathrm{A}$ the angular momentum of the magnetic star increases more than that of the corresponding non-magnetic star $\left(2 \times 10^{17} \mathrm{~cm}^{2} \mathrm{~s}^{-1}\right.$ for magnetic and $1.25 \times 10^{17} \mathrm{~cm}^{2} \mathrm{~s}^{-1}$ for non-magnetic star, $\sim 10^{4} \mathrm{yr}$ after fast Case A, at $3 M_{\odot}$ ).

The accretion stops when the secondary still has almost $50 \%$ of the hydrogen to burn in the core. Angular momentum is efficiently transported from the stellar core to the surface and the $\mu$-gradient can not stop it as in the case of the non-magnetic star. During further main sequence evolution,

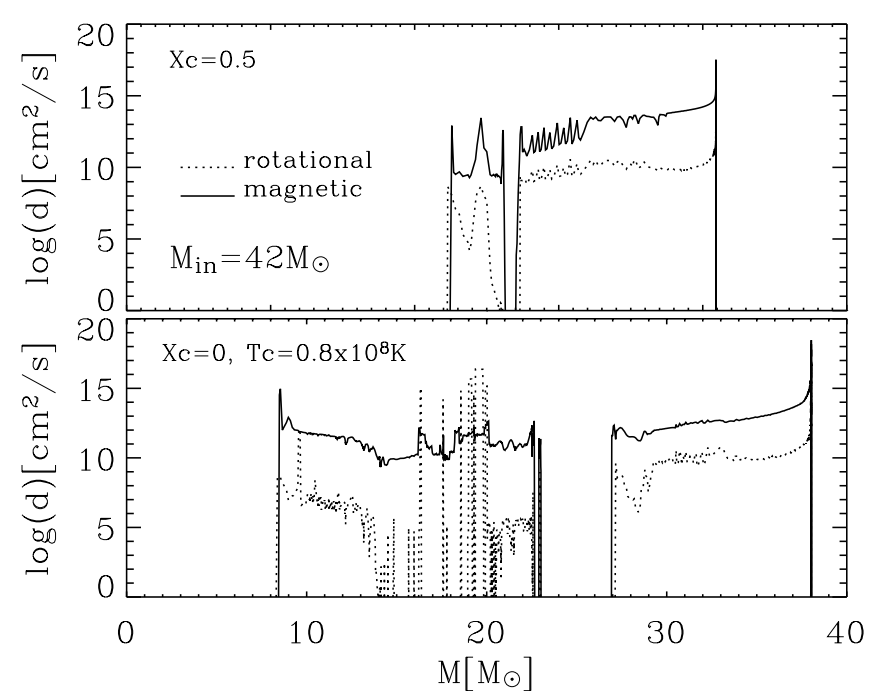

Fig. 17. Effective viscosities due to magnetic torques (solid line) and due to rotational instabilities (dotted line) for a rotating $42 M_{\odot}$ star at a central hydrogen abundance of $50 \%$ and at core hydrogen exhaustion.

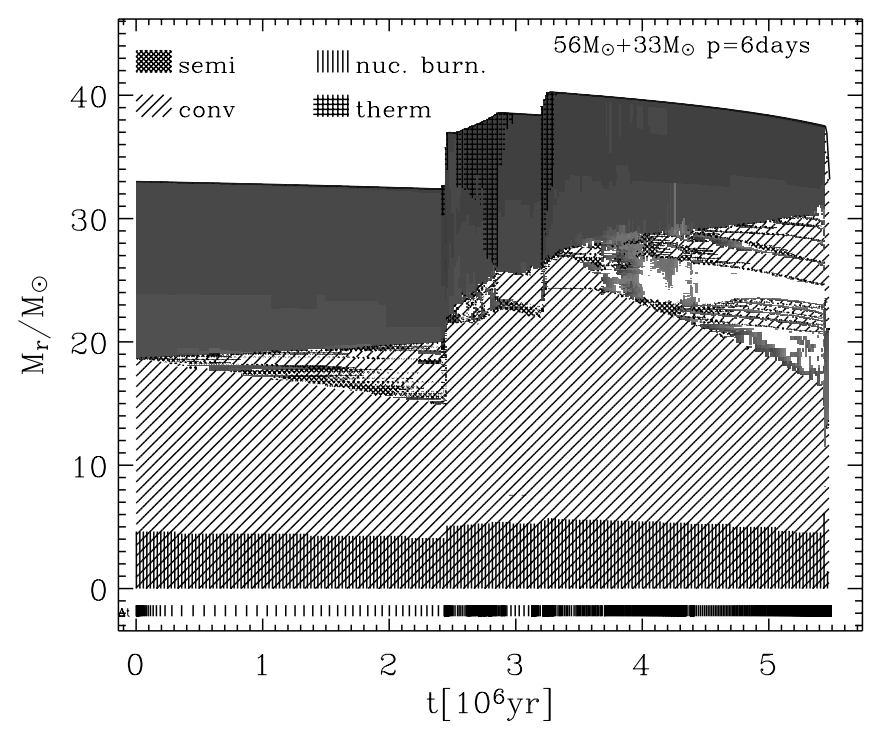

Fig. 18. Evolution of the internal structure of a rotating $33 M_{\odot}$ secondary star with magnetic field from the ZAMS until red supergiant phase $Y_{\mathrm{c}}=0.92$. See Fig. 7 for an explanation of the different hatching types.

the stellar core loses significant angular momentum and when hydrogen core burning stops, the specific angular momentum at $3 M_{\odot}$ is $2 \times 10^{16} \mathrm{~cm}^{2} \mathrm{~s}^{-1}$. Before helium ignites in the core, the specific angular momentum decreases to $6 \times 10^{15} \mathrm{~cm}^{2} \mathrm{~s}^{-1}$. The further evolution through the red supergiant phase slows down the core, since the envelope slows it down (magnetic viscosity can overcome the $\mu$-barrier).

Apparently, if a star with magnetic fields accretes matter half way in the main sequence evolution, it has enough time to lose angular momentum before hydrogen is exhausted in the core. This is why we compute models of non-magnetic and magnetic $33 M_{\odot}$ stars that accrete $2 M_{\odot}$ at the end of their main sequence evolution $\left(Y_{\mathrm{c}}=0.95\right)$. We assume an initial surface 


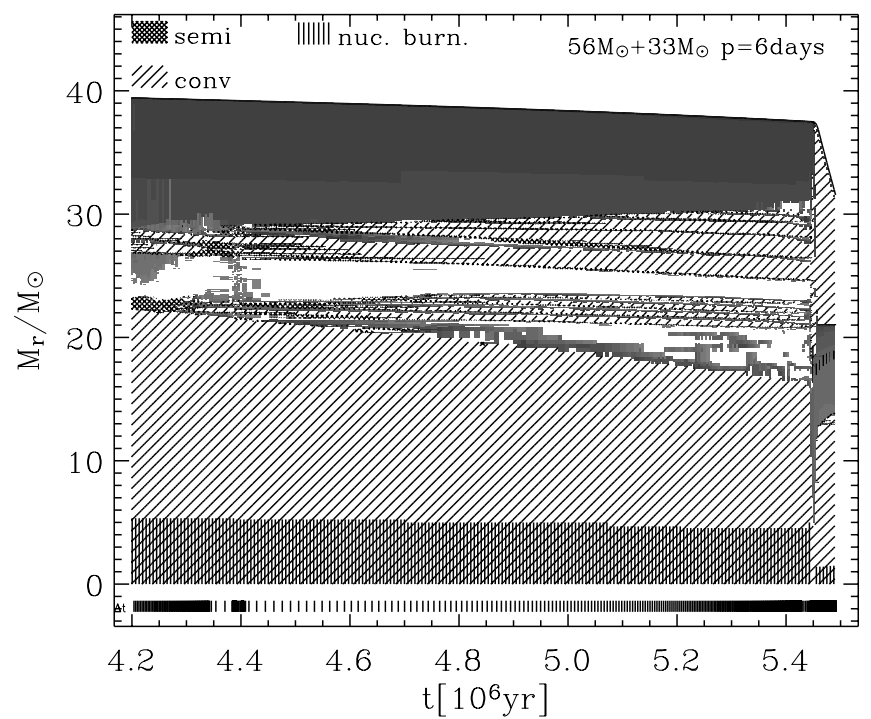

Fig. 19. Evolution of the internal structure of a rotating $33 M_{\odot}$ secondary star with magnetic field from after the $\mathrm{SN}$ explosion of the primary and disruption of the system. See Fig. 7 for an explanation of the different hatching types.

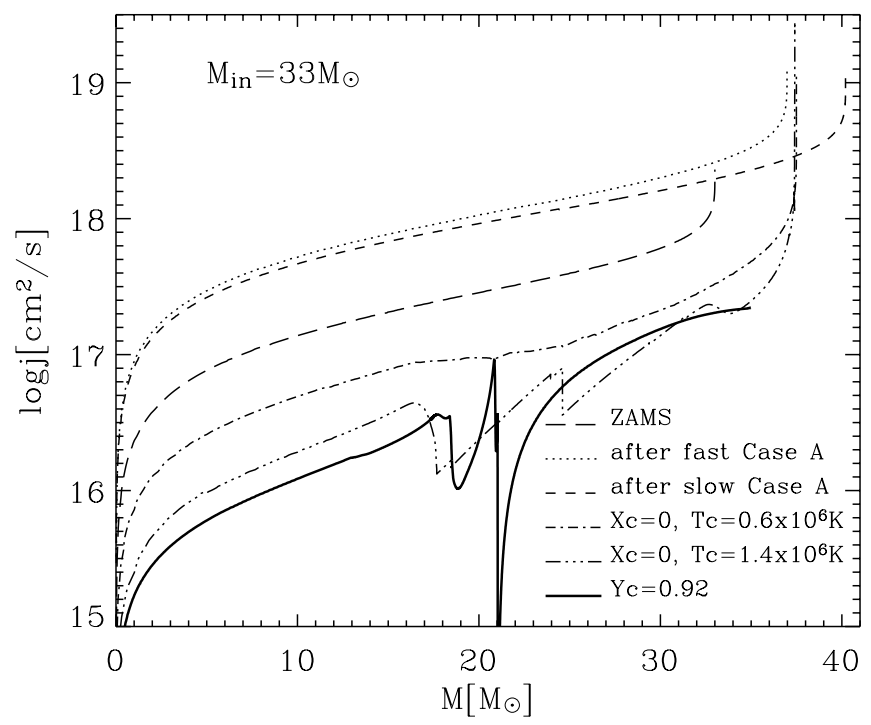

Fig. 20. Specific angular momentum profiles of the secondary star with magnetic fields on the hydrogen ZAMS (long dashed line), after fast (dotted line) and slow (short dashed line) Case A mass transfer, when all hydrogen is exhausted in the center (dot-dashed line), when helium ignites (three dots-dashed line) and and when the central helium abundance is $92 \%$ (solid line).

velocity of these stars of $\sim 60 \mathrm{~km} \mathrm{~s}^{-1}$ since they evolve in a binary system.

When the central helium abundance reaches $95 \%$, the core of the $33 M_{\odot}$ star with magnetic fields has a specific angular momentum of about $2 \times 10^{16} \mathrm{~cm}^{2} \mathrm{~s}^{-1}$ (Fig. 24, dotted line). On the other hand, the core of a star without magnetic fields hardly loses any angular momentum and its specific angular momentum is $4 \times 10^{16} \mathrm{~cm}^{2} \mathrm{~s}^{-1}$ (Fig. 23, dotted line). After this, both stars accrete $2 M_{\odot}$ of matter with an accretion rate of $10^{-4} M_{\odot} \mathrm{yr}^{-1}$. We assume that in this case stellar wind mass loss is not enhanced by rotation, so there is no additional

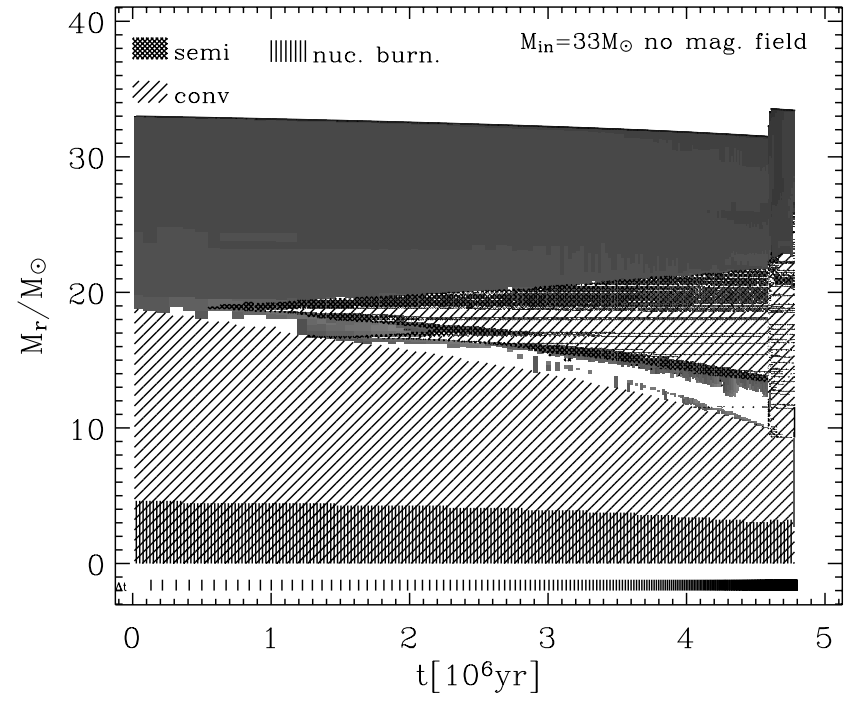

Fig. 21. Evolution of the internal structure of a rotating $33 M_{\odot}$ secondary star until helium ignition in the core. The star accretes $2 M_{\odot}$ when the central helium abundance is $95 \%$. See Fig. 7 for an explanation of the different hatching types.

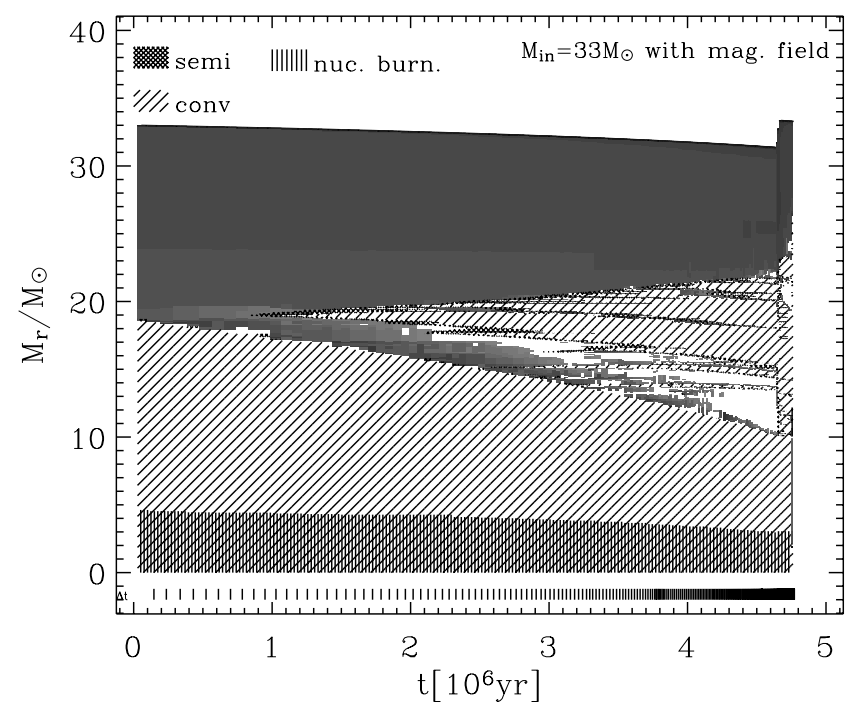

Fig. 22. Evolution of the internal structure of a rotating $33 M_{\odot}$ secondary star with magnetic field until helium ignition in the core. The star accretes $2 M_{\odot}$ when the central helium abundance is $95 \%$. See Fig. 7 for an explanation of the different hatching types.

angular momentum loss due to this effect. Accreted matter adds angular momentum to the surface layers and this angular momentum is then transported through the stellar interior much more efficiently in the model with magnetic field. At the end of accretion (Figs. 23 and 24, dashed line), angular momentum has been transported further inward in the magnetic star than in the non-magnetic star. If we look at the specific angular momentum profiles $10^{4} \mathrm{yr}$ after the accretion ended, we can notice that the specific angular momentum of the core of the magnetic star increases to almost $3 \times 10^{16} \mathrm{~cm}^{2} \mathrm{~s}^{-1}$ (at $3 M_{\odot}$ ). On the other hand, in the non-magnetic star angular momentum has been transported inward but still has not reached the core. 


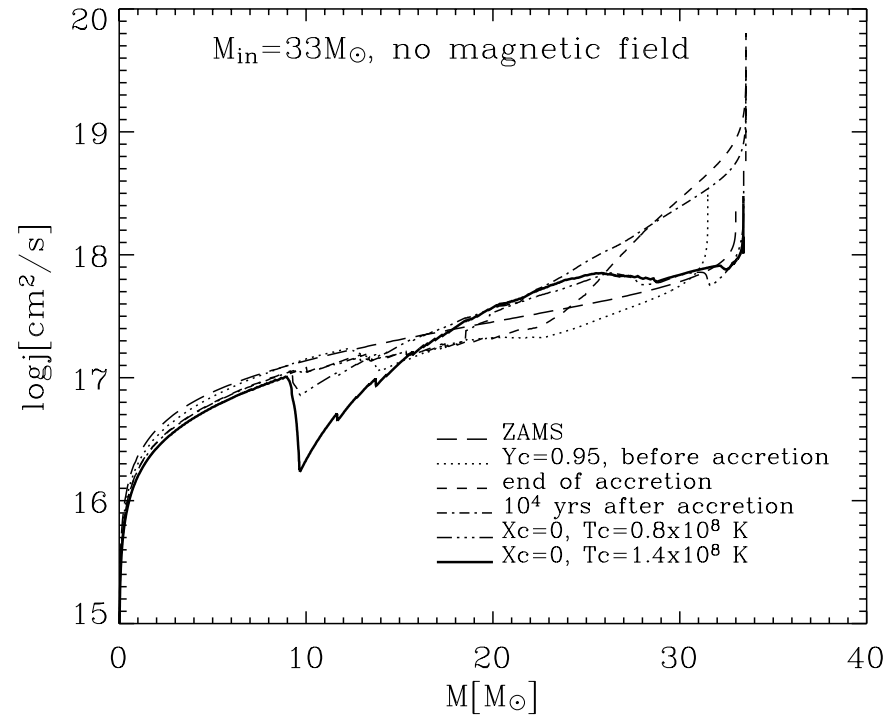

Fig. 23. Specific angular momentum profiles of a $33 M_{\odot}$ non-magnetic star on the ZAMS (long dashed line), when $Y_{\mathrm{c}}=95 \%$ (dotted line), at the end of the accretion (dashed line), $10^{4} \mathrm{yr}$ after the accretion (dotdashed line), when hydrogen is exhausted in the core (three dot-dashed line) and when helium ignites in the core (solid line).

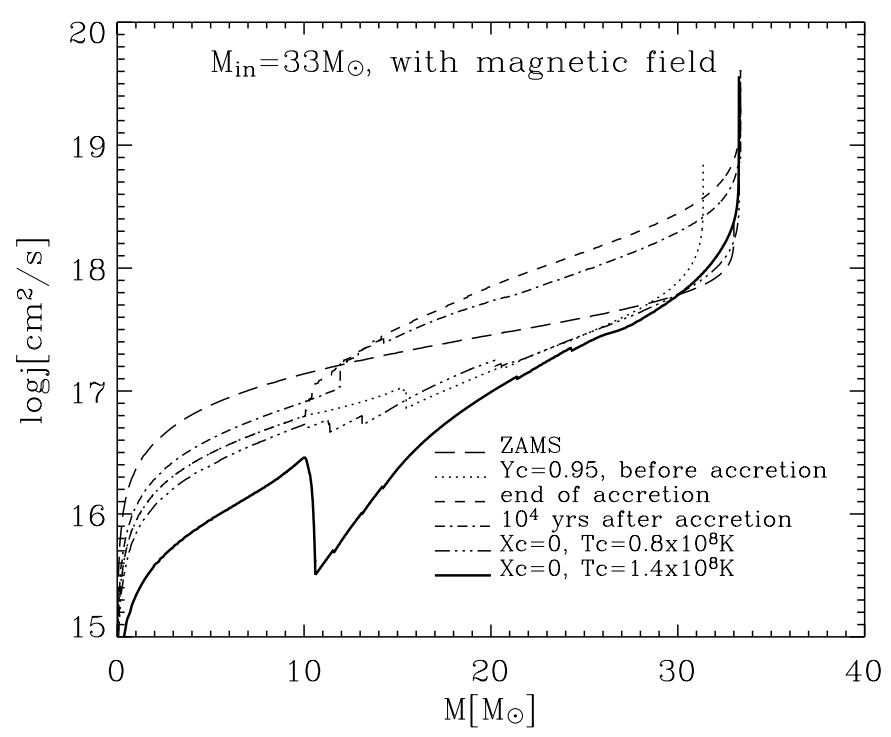

Fig. 24. Specific angular momentum profiles of a $33 M_{\odot}$ magnetic star on the ZAMS (long dashed line), when $Y_{\mathrm{c}}=95 \%$ (dotted line), at the end of the accretion (dashed line), $10^{4} \mathrm{yr}$ after the accretion (dotdashed line), when hydrogen is exhausted in the core (three dot-dashed line) and when helium ignites in the core (solid line).

The magnetic star continues to lose angular momentum from the core during the remaining main sequence evolution $\left(\sim 10^{5} \mathrm{yr}\right)$, and when hydrogen is exhausted in the core the specific angular momentum at $3 M_{\odot}$ is $1.6 \times 10^{16} \mathrm{~cm}^{2} \mathrm{~s}^{-1}$. As explained above, in the magnetic model, the core loses significant amounts of angular momentum between core hydrogen exhaustion and core helium ignition, and when central helium burning starts, the specific angular momentum of the core at $3 M_{\odot}$ is only $6 \times 10^{15} \mathrm{~cm}^{2} \mathrm{~s}^{-1}$.

\section{Conclusions}

The single star models presented above confirm that nonmagnetic stars in the mass range relevant for GRB production through collapsars may retain enough core angular momentum for a GRB to form. In particular, the stars that evolve into red supergiants and become Wolf-Rayet stars only at the end of core helium burning can avoid a significant core angular momentum loss through Wolf-Rayet winds and may retain a specific angular momentum of up to $10^{17} \mathrm{~cm}^{2} \mathrm{~s}^{-1}$ in their iron cores. In these models, an effective core angular momentum loss during the main sequence is prevented by the suppression of rotational mixing in regions containing a mean molecular weight gradient. Angular momentum loss from the stellar core during helium burning is insignificant. Contrary to the trend found in Heger et al. (2000a), we find that a $42 M_{\odot}$ star may end its life with a larger specific core angular momentum than a $20 M_{\odot}$. The reason is that, for the same initial equatorial rotation velocity, the initial specific angular momentum in the $42 M_{\odot}$ is significantly larger due to its larger initial radius. We note that a similar trend has been found by Hirschi et al. (2004).

The dynamo model of Spruit (2002) cause a significant angular momentum transport even in the presence of mean molecular weight gradients. Heger et al. (2004a) already found the increased coupling of core and envelope to result in iron core specific angular momenta of the order of $10^{14} \mathrm{~cm}^{2} \mathrm{~s}^{-1}$ for stars between 10 and $25 M_{\odot}$. Our magnetic $42 M_{\odot}$ single star model was only computed up to core helium ignition. By then, however, the specific core angular momentum was already reduced by a factor of 30 from the initial value. Heger et al. (2004a) showed that during core helium burning the core-envelope coupling reduces the core angular momentum by another order of magnitude. The final specific core angular momentum in our $42 M_{\odot}$ star can thus be estimated to fall below $\sim 10^{15} \mathrm{~cm}^{2} \mathrm{~s}^{-1}$, which will render effects of rotation during the core collapse insignificant.

We also model the evolution of a $56 M_{\odot}+33 M_{\odot}$ binary system with an initial orbital period of 6 days. Since the binaryenhanced mass loss of the primary leads to extremely slow rotation, our attention focuses on the secondary star, which is spun-up due to accretion of mass and angular momentum. We computed two binary evolution sequences, with and without magnetic fields. In both sequences, the initial $33 M_{\odot}$ star grows to about $40 M_{\odot}$ due to accretion during its main sequence evolution, and subsequently evolves into a red supergiant. Before the mass transfer, tidal spin-orbit coupling leads to bound rotation of both stars and to a specific angular momentum which is a factor of $3 . . .5$ smaller than in a corresponding single star.

The accretion leads to a significant spin-up of the star. In the non-magnetic model, the mean molecular weight gradient limits the inward diffusion of angular momentum, and the corresponding spin-up of the core merely compensates the tidally induced angular momentum loss. The result is a helium core that rotationally decouples from the envelope with roughly the same mass and angular momentum as in the case of a $42 M_{\odot}$ single star, i.e., with high enough specific angular momentum to produce a GRB. 
In the magnetic model, the core spin-up due to accretion is stronger. It temporarily leads to a core spin rate which is factor of $2 \ldots 3$ above that of a ZAMS star of comparable mass. Magnetic core-envelope coupling, however, has reduced the specific core angular momentum by almost a factor 100 by the time the star has started core helium burning. Its final core angular momentum will thus be comparable to that of the magnetic $42 M_{\odot}$ single star model discussed above: probably too small to produce a GRB.

Clearly, the effect of core spin-up due to accretion on the final core angular momentum will be larger if the accretion occurs later in the evolution of the accretion star, as there will be less time to lose again the angular momentum gained by accretion. Therefore, we performed the numerical experiment of accreting $2 M_{\odot}$ of material on a $33 M_{\odot}$ at a time when the core hydrogen concentration was down to $5 \%$ (instead of about $40 \%$ in the binary evolution model). The result was again that the core-envelope coupling in the magnetic model was overwhelming and prevents this scenario to be a realistic option for GRB production.

We conclude that our binary models without magnetic field can reproduce stellar cores with a high enough specific angular momentum $\left(j \geq 3 \times 10^{16} \mathrm{~cm}^{2} \mathrm{~s}^{-1}\right)$ to produce a collapsar and a GRB.

If magnetic field is taken into consideration, however, GRBs at near solar metallicity need to be produced in rather exotic binary channels, or the magnetic effects are overestimated in our current models. The first option is not implausible, since reverse mass transfer from the original secondary star onto the primary star during its Wolf-Rayet phase (Wellstein et al. 2001), or late stellar merger may lead to an efficient core spinup. The realization frequency of such events, however, even though it is difficult to estimate, may still be small. The latter would require a significant angular momentum loss from the iron core, either during collapse or from the proto-neutron star, in order to explain the relatively slow rotation rates of young pulsars (cf., however, Heger et al. 2004b).

Acknowledgements. A.H. has been supported under the auspices of the US Department of Energy by its contract W-7405-ENG-36 to the Los Alamos National Laboratory, by DOE SciDAC grant DE-FC02-01ER41176, and by NASA grant SWIF03-0047-0037.

\section{References}

Braun, A. 1998, Ph.D. Thesis

Braun, H., \& Langer, N. 1995, A\&A, 297, 483
Brookshaw, L., \& Tavani, M. 1993, ApJ, 410, 719

Fryer, C. L. 1999, ApJ, 522, 413

Fukuda, I. 1982, PASP, 94, 271

Hamann, W.-R., \& Koesterke, L. 1998, A\&A, 335, 1003

Hamann, W.-R., Koesterke, L., \& Wessolowski, U. 1995, A\&A, 299, 151

Heger, A., Langer, N., \& Woosley, S. E. 2000a, ApJ, 528, 368

Heger, A., Woosley, S. E., \& Langer, N. 2000b, New Astron. Rev., 44, 297

Heger, A., Woosley, S. E., Langer, N., \& Spruit, H. C. 2004a, in IAU Symp., 215

Heger, A., Woosley, S. E., \& Spruit, H. C. 2004b, [arXiv: astro-ph/0409422]

Hirschi, R., Meynet, G., \& Maeder, A. 2004, A\&A, 425, 649

Hjorth, J., Sollerman, J., Møller, P., et al. 2003, Nature, 423, 847

Iglesias, C. A., \& Rogers, F. J. 1996, ApJ, 464, 943

King, A. R., Schenker, K., Kolb, U., \& Davies, M. B. 2001, MNRAS, 321,327

Kippenhahn, R., \& Thomas, H.-C. 1970, in Stellar Rotation, IAU Coll., 4, 20

Kopal, Z. 1978, Dynamics of Close Binary Systems

Kudritzki, R. P., Pauldrach, A., Puls, J., \& Abbott, D. C. 1989, A\&A, 219, 205

Langer, N. 1991, A\&A, 252, 669

Langer, N. 1998, A\&A, 329, 551

MacFadyen, A. I., \& Woosley, S. E. 1999, ApJ, 524, 262

Meyer, F., \& Meyer-Hofmeister, E. 1983, A\&A, 121, 29

Meynet, G., \& Maeder, A. 1997, A\&A, 321, 465

Nieuwenhuijzen, H., \& de Jager, C. 1990, A\&A, 231, 134

Noels, A., \& Grevesse, N. 1993, in Inside the Stars, ASP Conf. Ser., 40, IAU Coll., 137, 410

Petrovic, J., Langer, N., \& vanderHucht, K. 2004, A\&A, submitted

Pinsonneault, M. H., Kawaler, S. D., Sofia, S., \& Demarque, P. 1989, ApJ, 338, 424

Podsiadlowski, P., Joss, P. C., \& Hsu, J. J. L. 1992, ApJ, 391, 246

Price, P. A., Berger, E., Reichart, D. E., et al. 2002, ApJ, 572, L51

Ritter, H. 1988, A\&A, 202, 93

Spruit, H. C. 2002, A\&A, 381, 923

Spruit, H. C., \& Phinney, E. S. 1998, Nature, 393, 139

van Marle, A.-J., Langer, N., \& Garcia-Segura, G. 2004, A\&A, in prep.

Wellstein, S. 2001, Ph.D. Thesis

Wellstein, S., \& Langer, N. 1999, A\&A, 350, 148

Wellstein, S., Langer, N., \& Braun, H. 2001, A\&A, 369, 939

Woosley, S. E. 1993a, BAAS, 25, 894

Woosley, S. E. 1993b, ApJ, 405, 273

Zahn, J.-P. 1977, A\&A, 57, 383 